\title{
Carotenoid Composition of the Fruit of Red Mamey (Pouteria sapota)
}

Enrique Murillo, Erika Turcsi, Ildikó Szabó, Yesuri Mosquera, Attila Agócs, Veronika Nagy,

Gergely Gulyás-Fekete, József Deli

\section{Supporting Information}

\section{Structural formulas of the carotenoids}

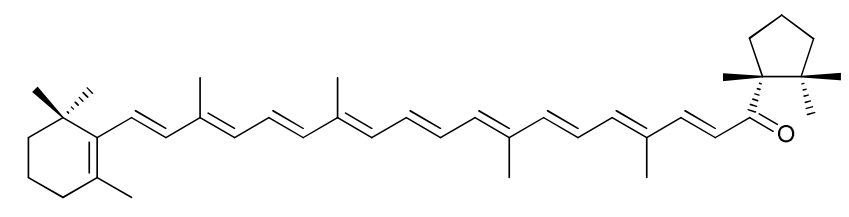

Sapotexanthin 1

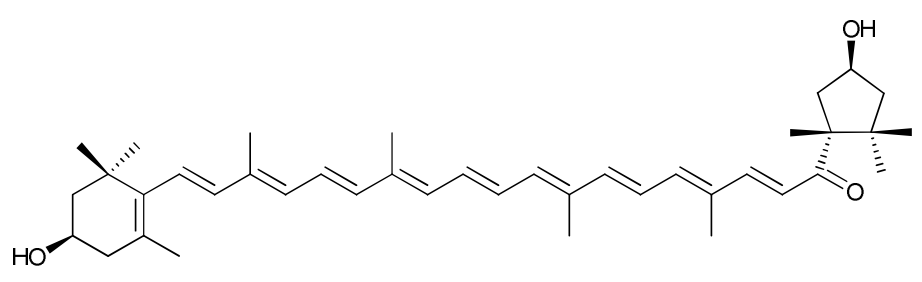

Capsanthin 2

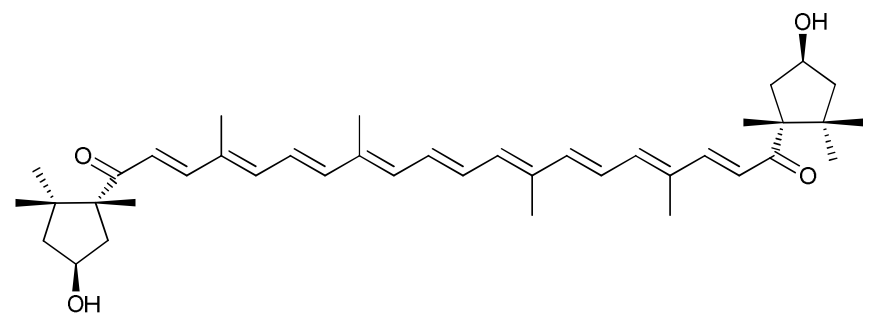

Capsorubin 3

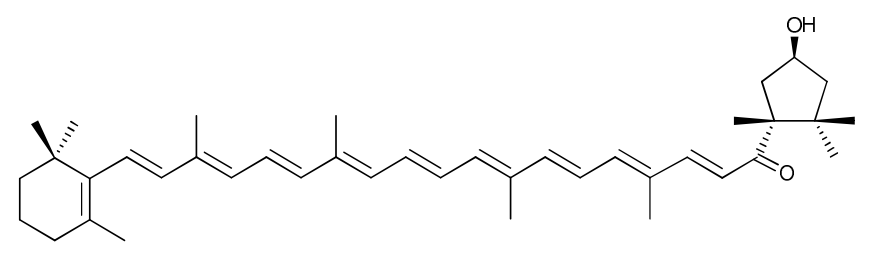




\section{Cryptocapsin 4}

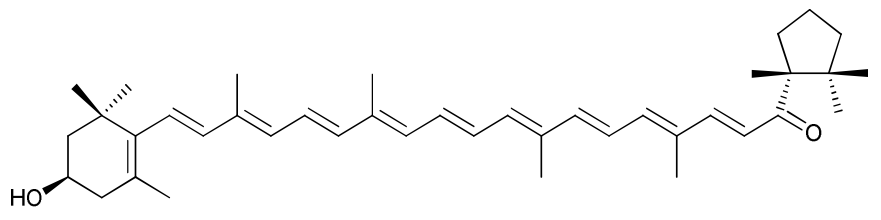

3'-Deoxycapsanthin $\mathbf{5}$

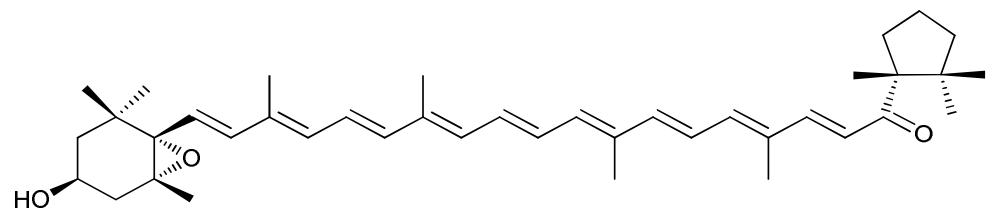

3'-Deoxycapsanthin 5,6-epoxide 6

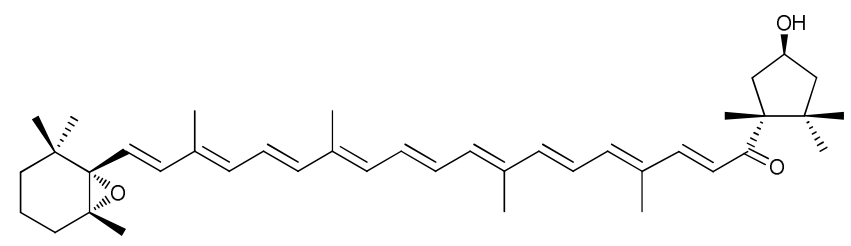

Cryptocapsin 5,6-epoxide 7

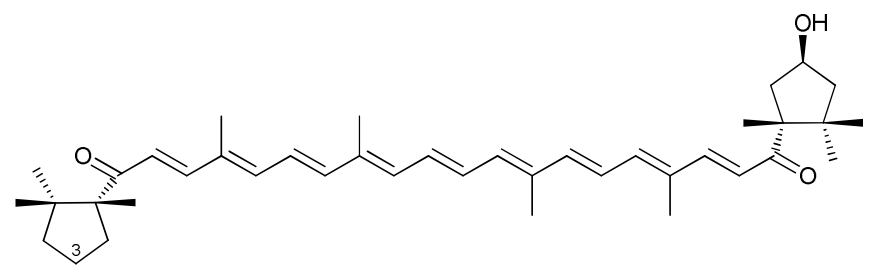

3-Deoxycapsorubin 8

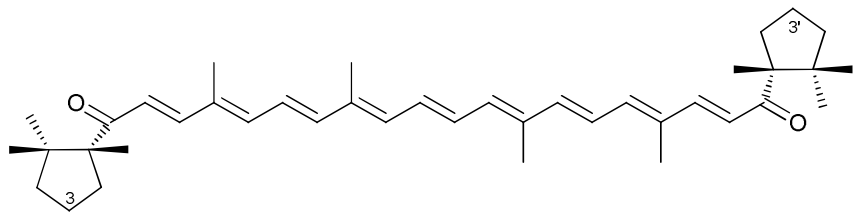

\section{3,3'-Dideoxycapsorubin 9}

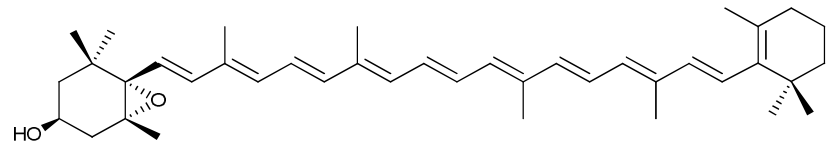

$\beta$-Cryptoxanthin 5,6-epoxide 10 


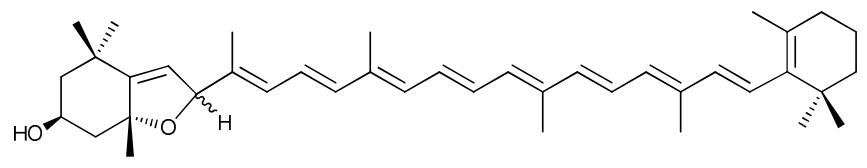

$\beta$-Cryptoxanthin 5,8-epoxide 11

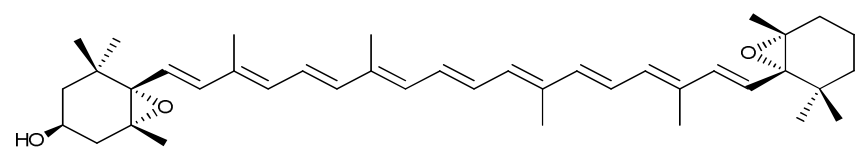

$\beta$-Cryptoxanthin 5,6,5',6'-diepoxide 12

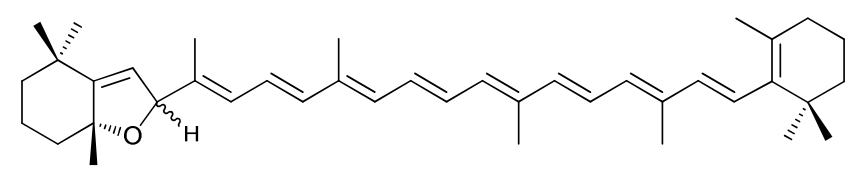

$\beta$-Carotene 5,8-epoxide 13

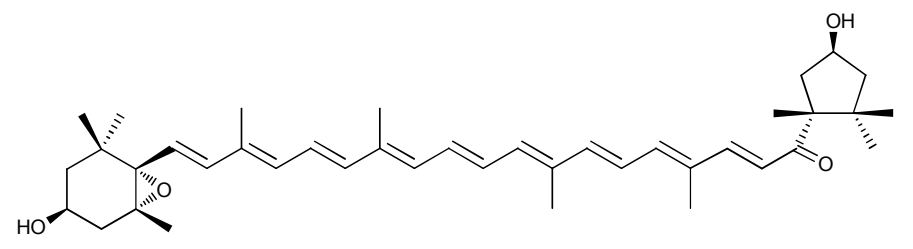

Capsanthin 5,6-epoxide 14

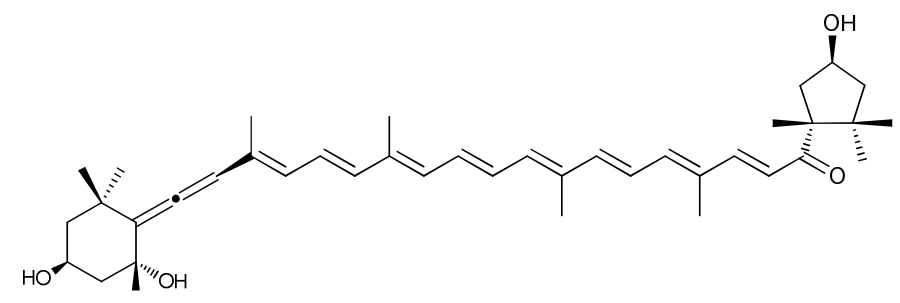

Capsoneoxanthin 15

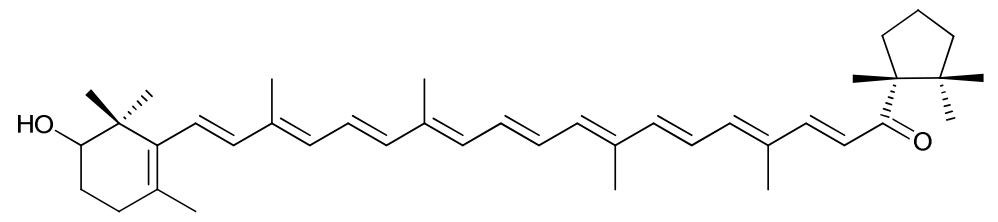

Iso-3'-Deoxycapsanthin 16

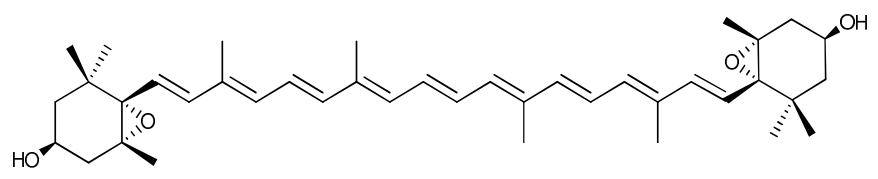

Violaxanthin 17 


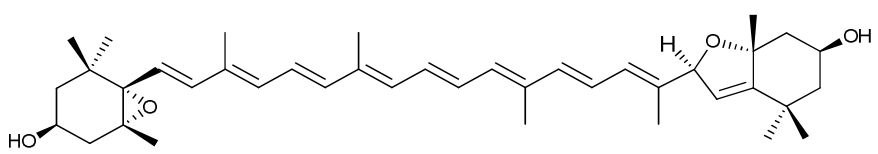

(5'R, 8'S)-Luteoxanthin 18<smiles>CC(/C=C/C=C(\C)C1C=C2C(C)(C)CC(O)C[C@]2(C)O1)=C\C=C\C=C\C(C)=C\C=C\C(C)=C\C=C\[C@@H]1[C@@H](C)C[C@@H](O)CC1(C)C</smiles>

$\left(5^{\prime} R, 8^{\prime} R\right)$-Luteoxanthin 19

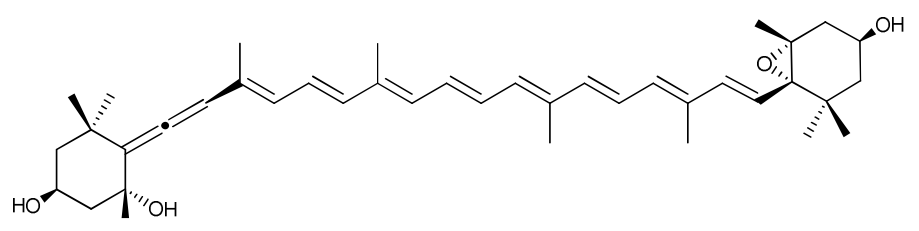

Neoxanthin 20

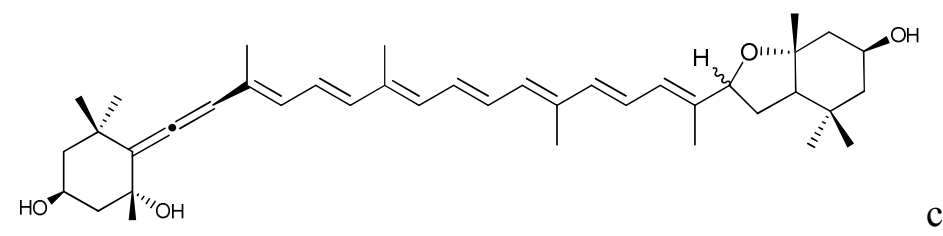

Neochrome 21

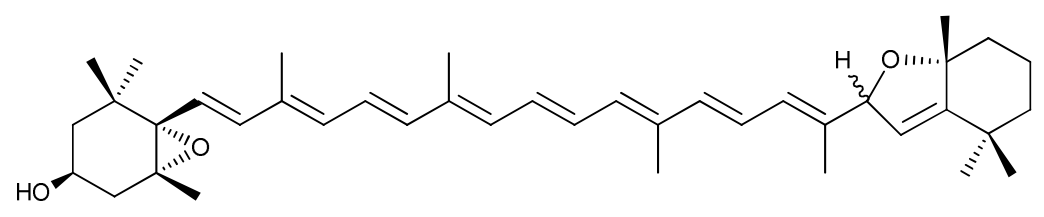

$\beta$-Cryptoxanthin 5,6,5',8'-diepoxide 22<smiles>CC(/C=C/C=C(C)/C=C/[C@@]1(C)[C@@H](C)CCCC1(C)C)=C\C=C\C=C(C)\C=C\C=C(/C)[C@H]1C=C2[C@@H](C)CC(O)C[C@]2(C)O1</smiles>

$\beta$-Cryptoxanthin 5,8,5',6'-diepoxide 23

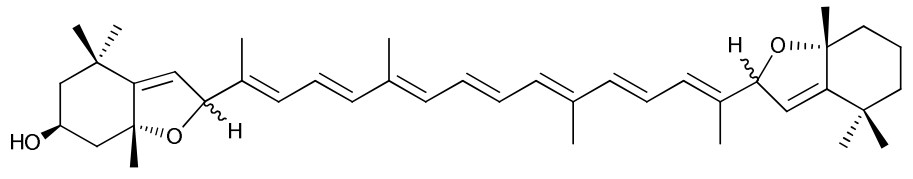

$\beta$-Cryptoxanthin 5,8,5'8'-diepoxide $\mathbf{2 4}$ 


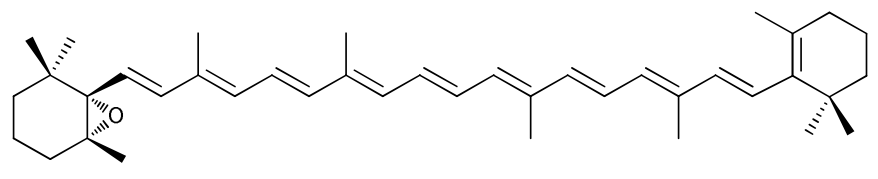

$\beta$-Carotene 5,6-epoxide 25

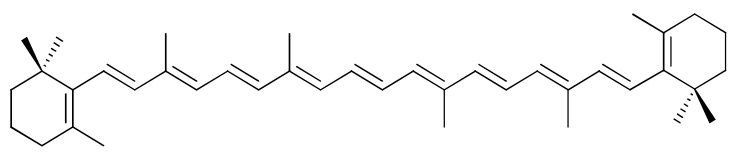

$\beta$-Carotene 26

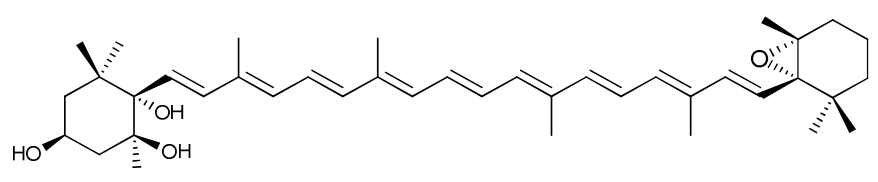

5,6-Diepilatoxanthin 27

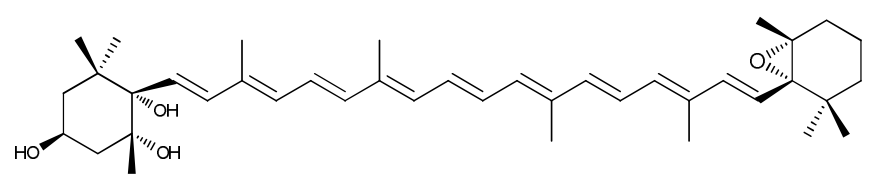

6-Epilatoxanthin 28

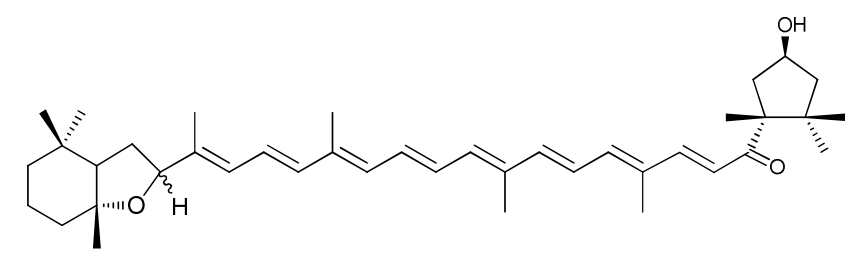

Cryptocapsin 5,8-epoxide 29

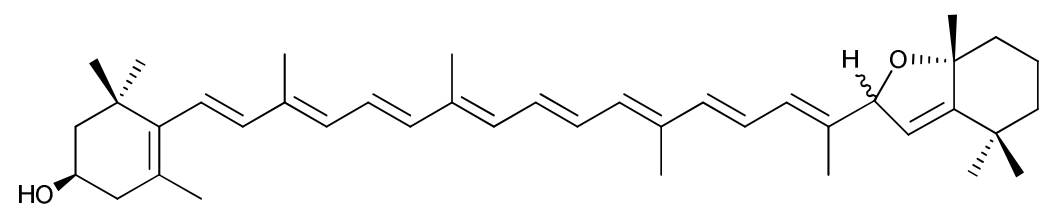

$\beta$-Cryptoxanthin 5', $8^{\prime}$-epoxide 30

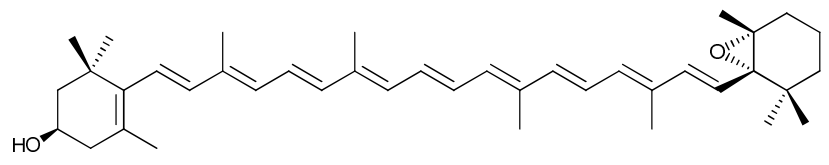

$\beta$-Cryptoxanthin 5',6'-epoxide 31 


\section{MS spectra of some identified carotenoids}

Peak 8: Neoxanthin Mw: 600

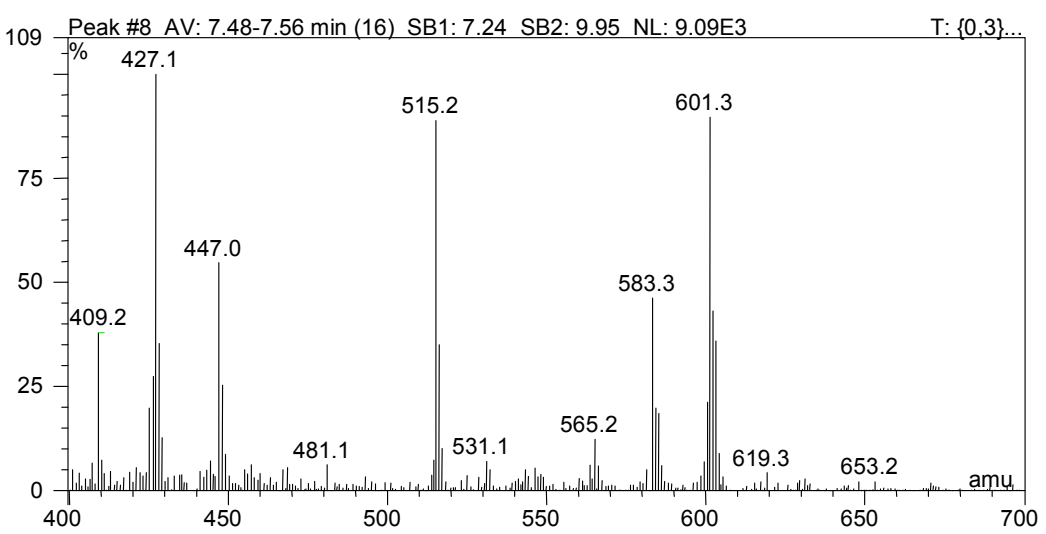

Peak 10: Violaxanthin Mw: 600

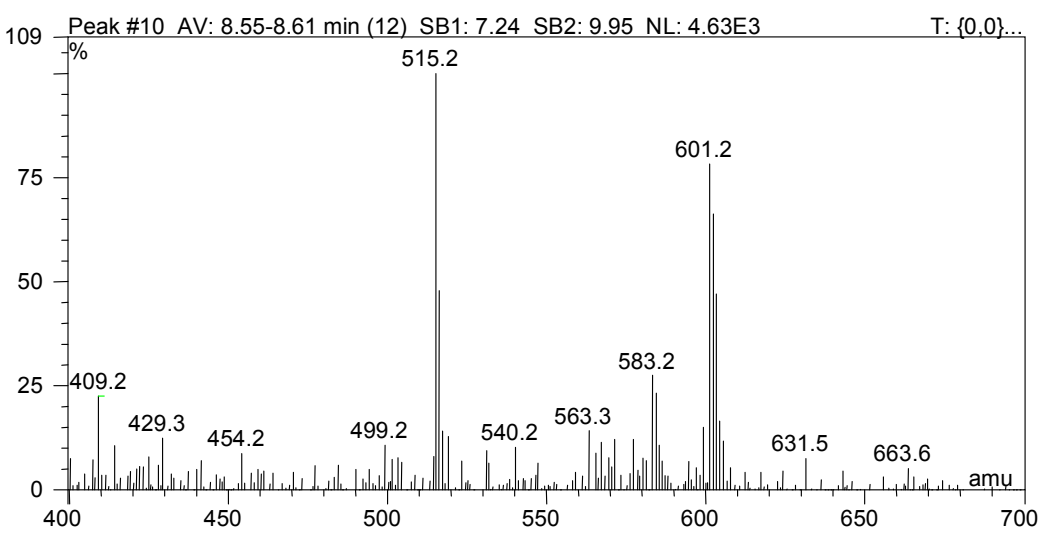

Peak 12: Capsoneoxanthin Mw: 600

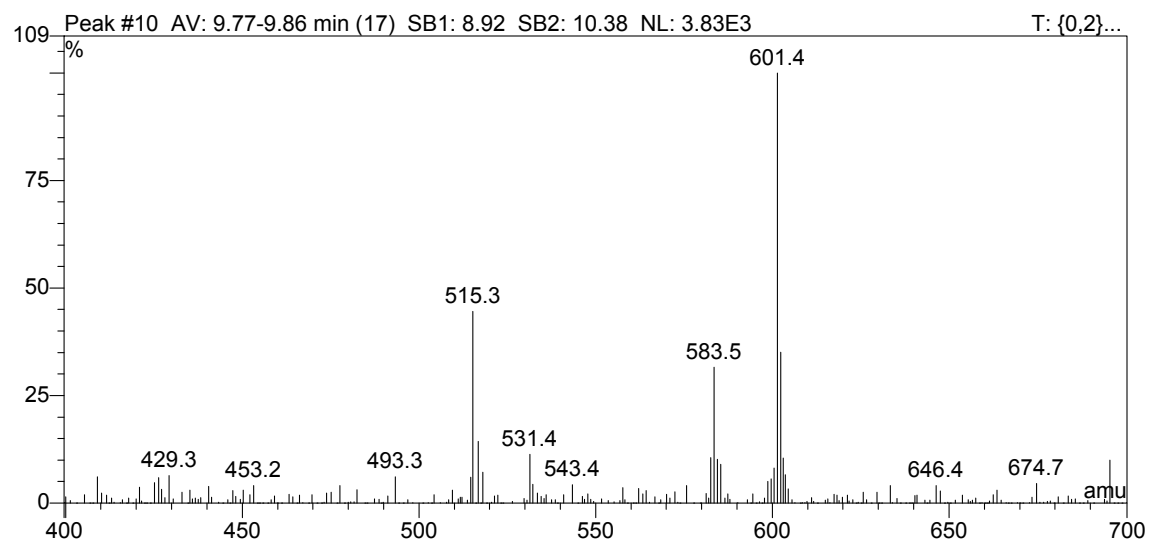


Peak 13: Luteoxanthin Mw: 600

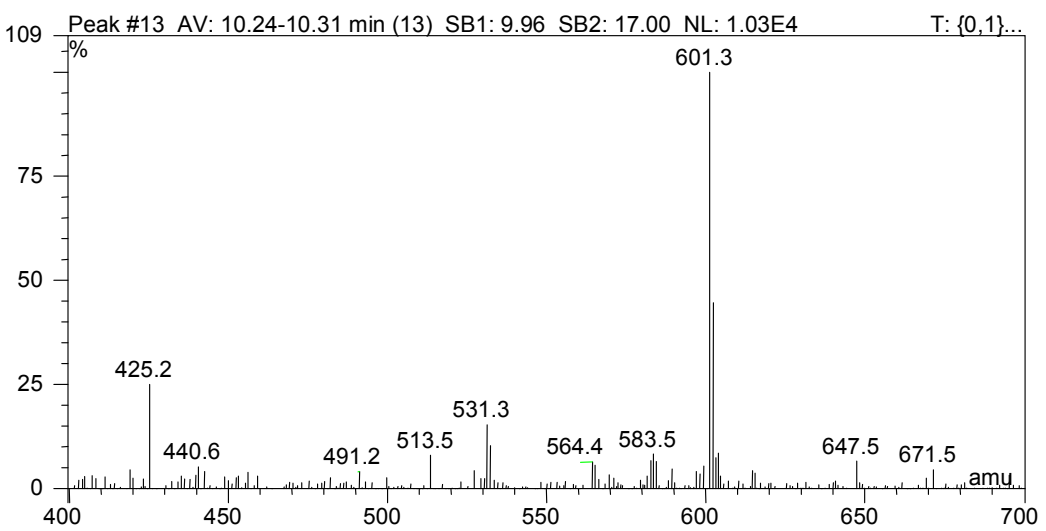

Peak 14. Capsanthin 5,6-epoxide Mw: 600

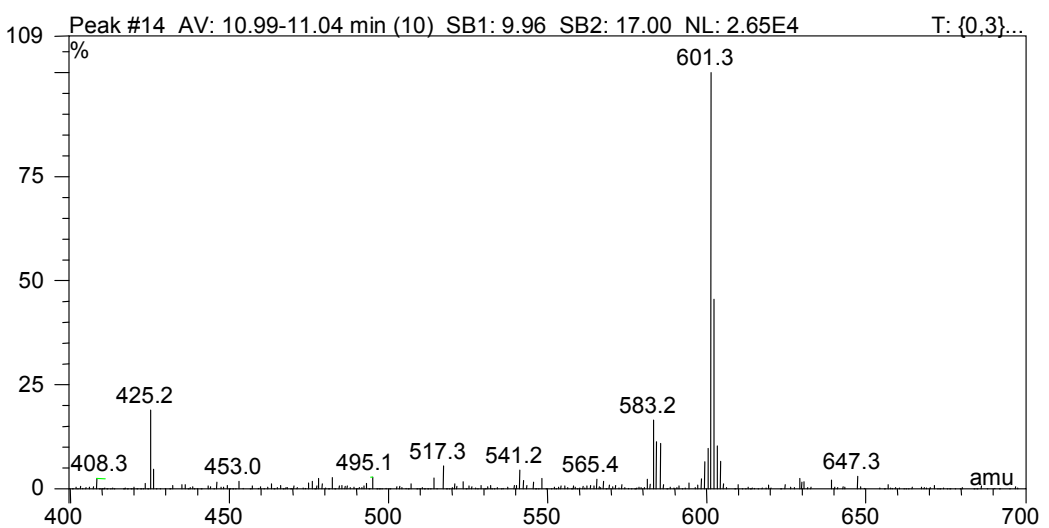

Peak 15: $\beta$-Cryptoxanthin 5,6,5',6'-diepoxide Mw: 584

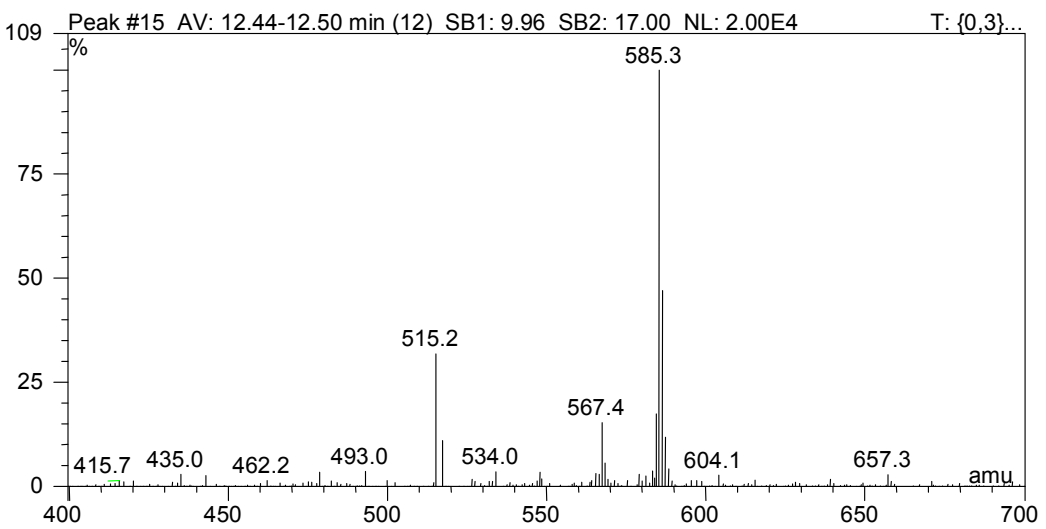


Peak 16: (9Z)-Violaxanthin Mw: 600

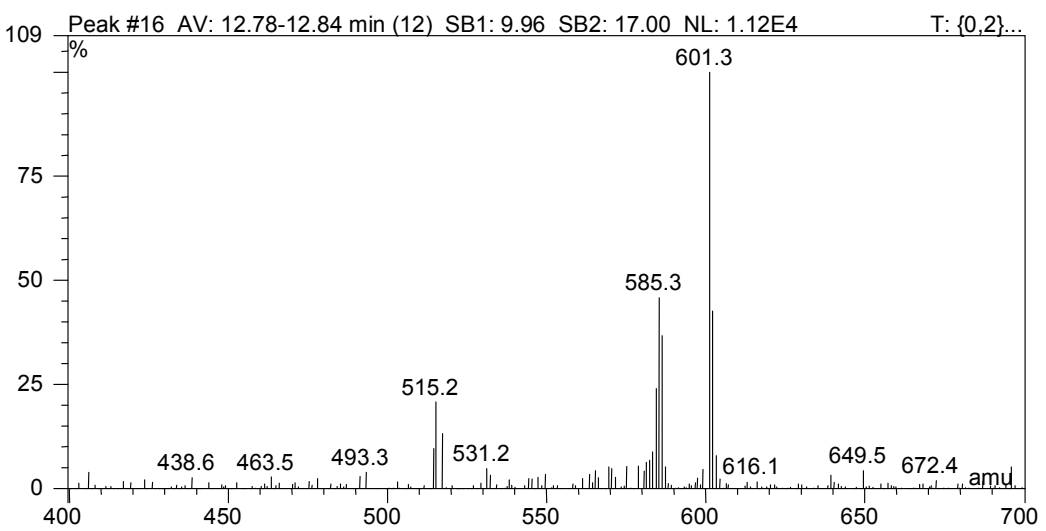

Peak 19: $\beta$-Cryptoxanthin 5,6,5',8'-epoxide Mw:584

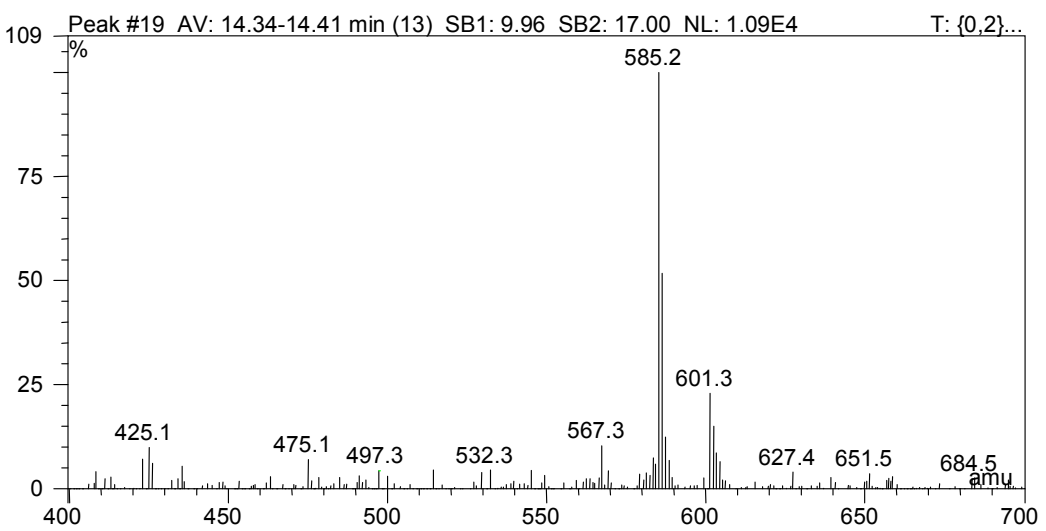

Peak 22: Cryptocapsin 5,6-epoxide Mw: 584

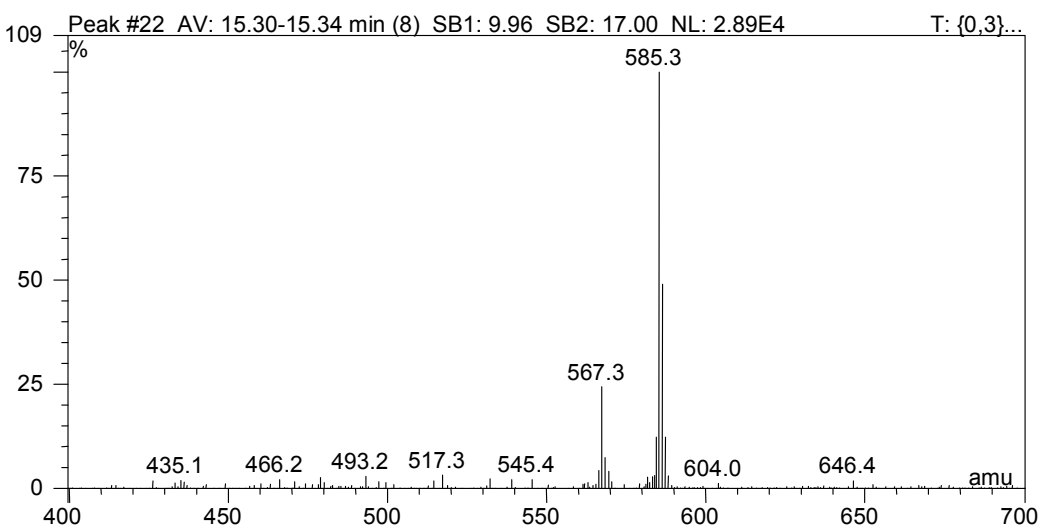


Peak 32 (13Z)-Cryptocapsin Mw: 568

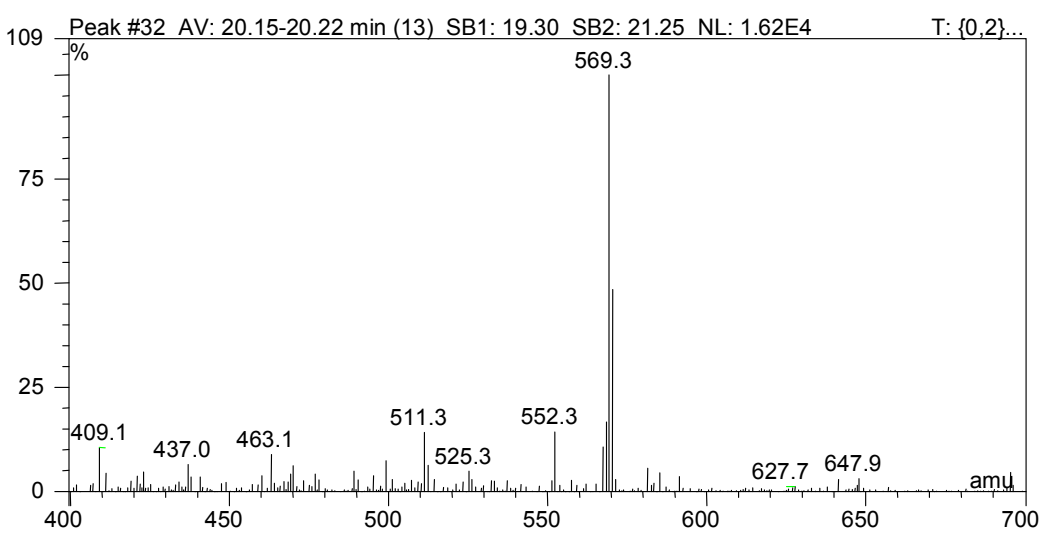

Peak 37: $\beta$-Cryptoxanthin 5,8-epoxide Mw: 568

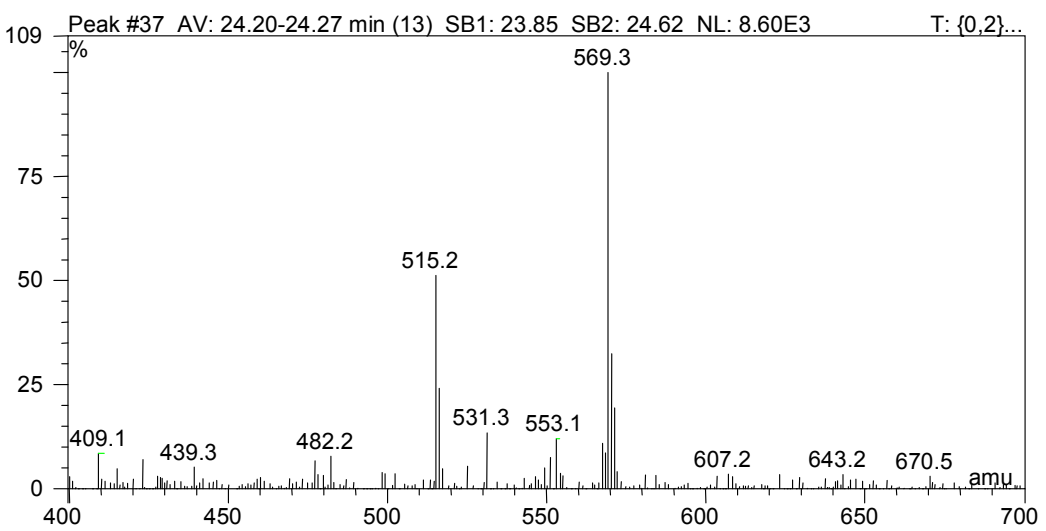

Peak 40: Cryptocapsin Mw: 568

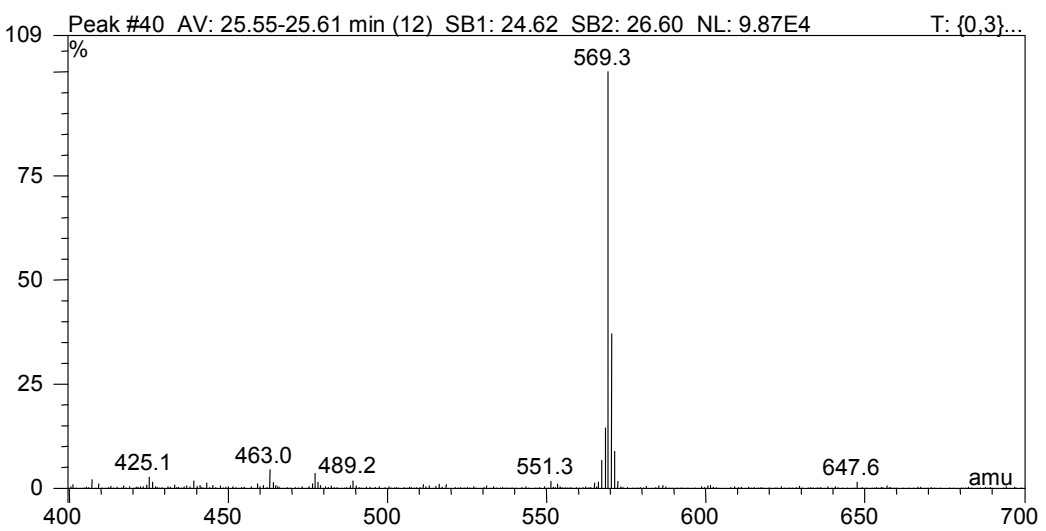


Peak 41: (13/13'Z)-Sapotexanthin Mw:552

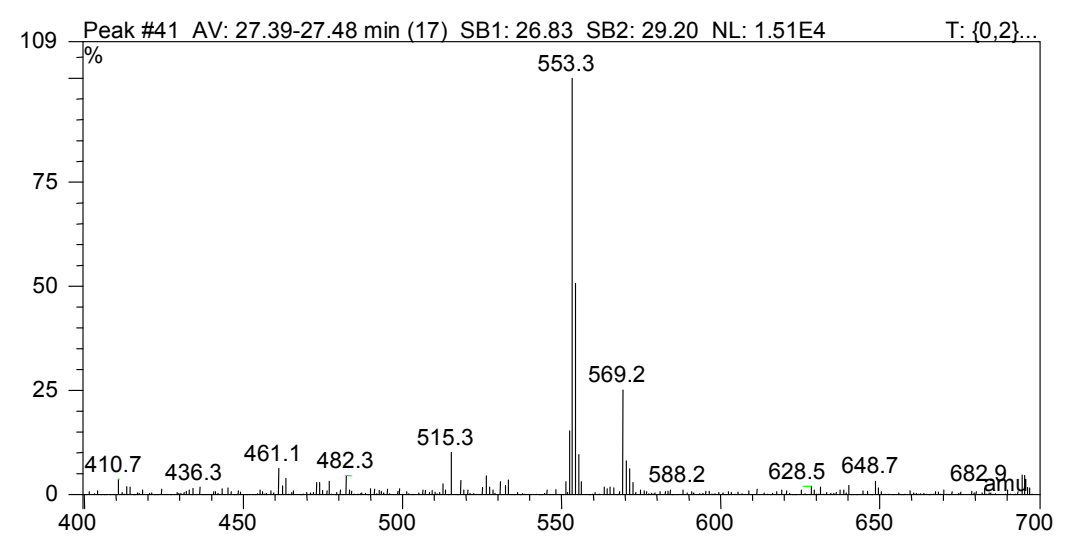

Peak 44: $\beta$-Carotene 5,8-epoxide Mw: 552

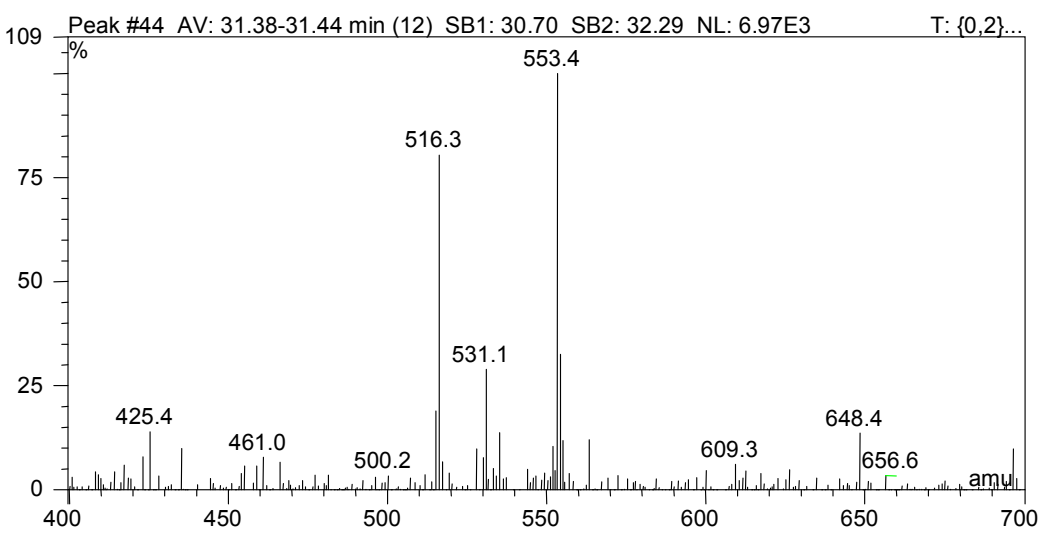

\section{Peak 45: Sapotexanthin Mw: 552}

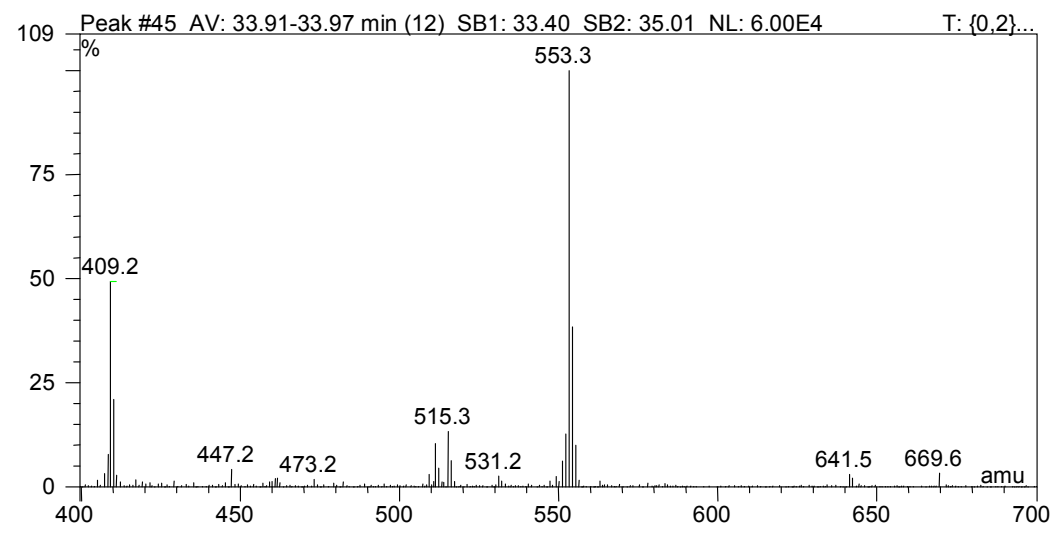




\section{UV-Vis spectra of some identified carotenoids (solvent: benzene)}

Peak 1: Unidentified

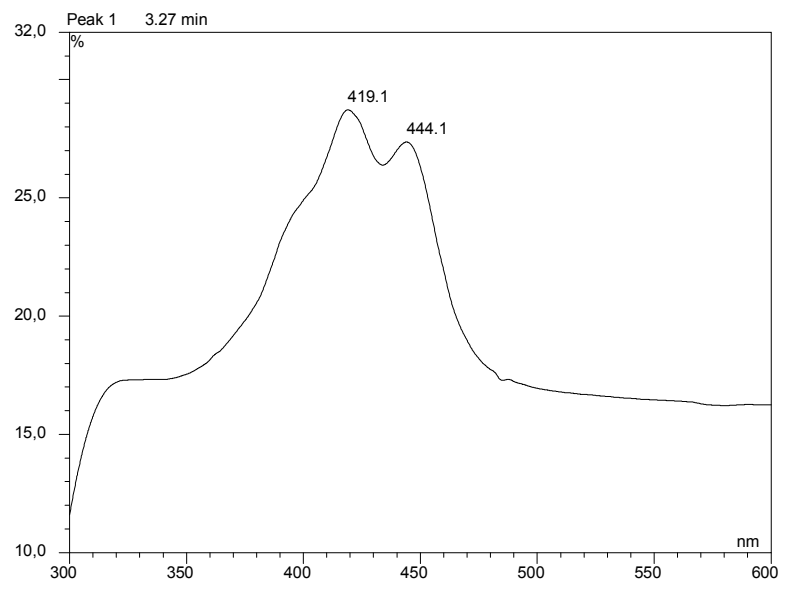

Peak 2: Unidentified

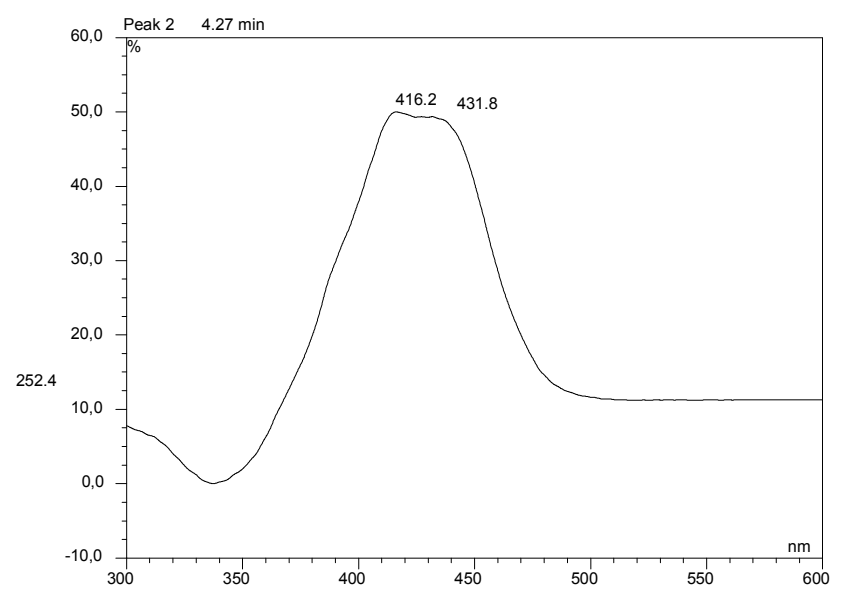

Peak 3: Unidentified

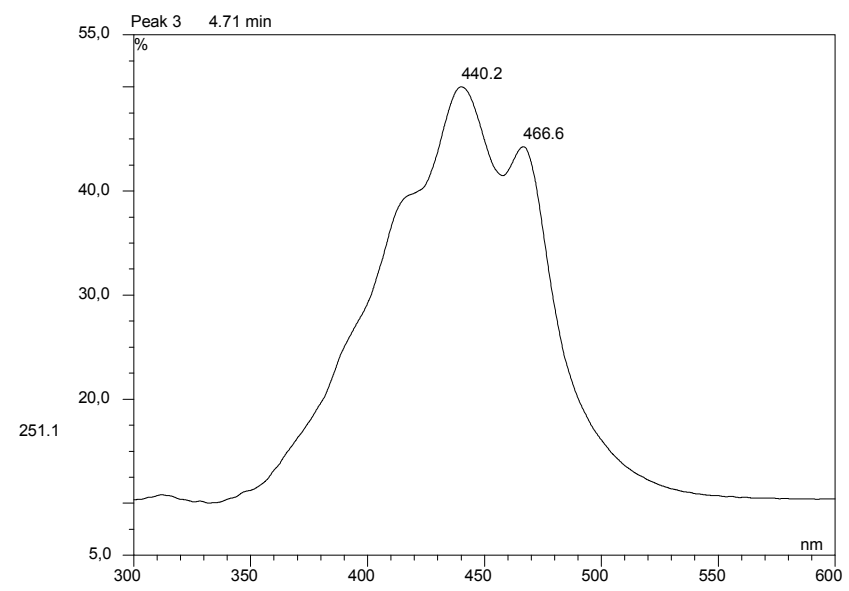


Peak 4: 5,6-Diepilatoxanthin

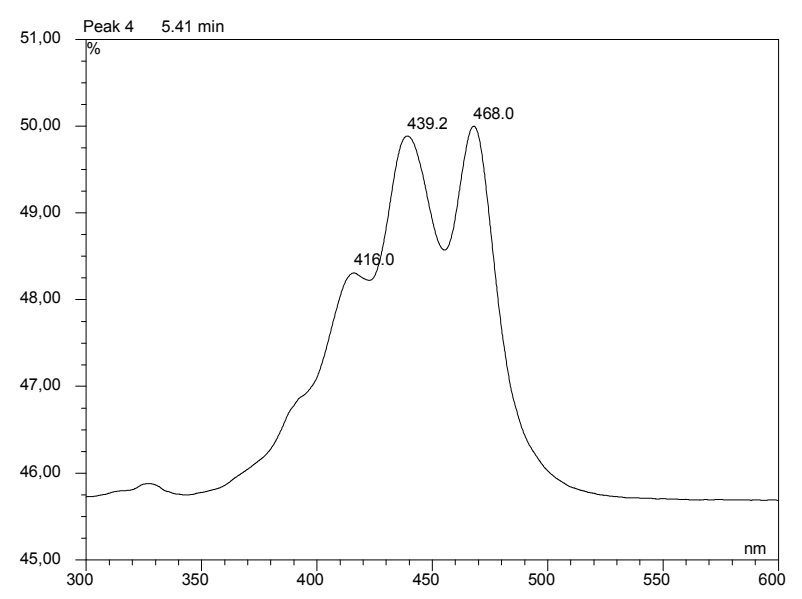

Peak 5: 6-Epilatoxanthin

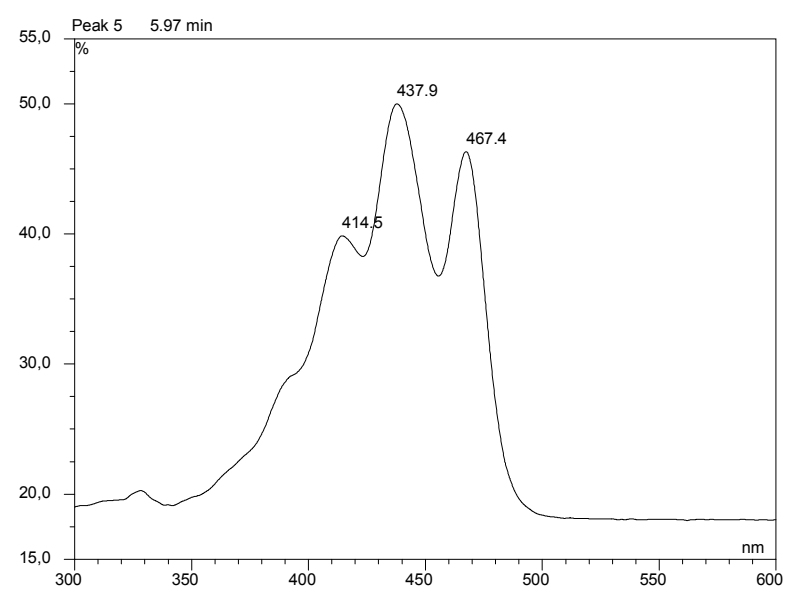

Peak 6: Unidentified

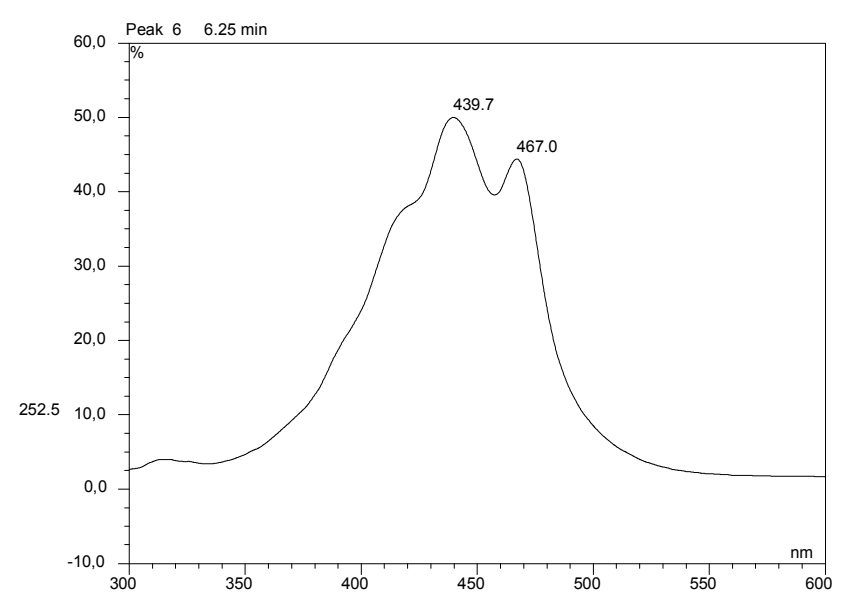


Peak 7: (9/9'Z)-5,6-Diepilatoxanthin

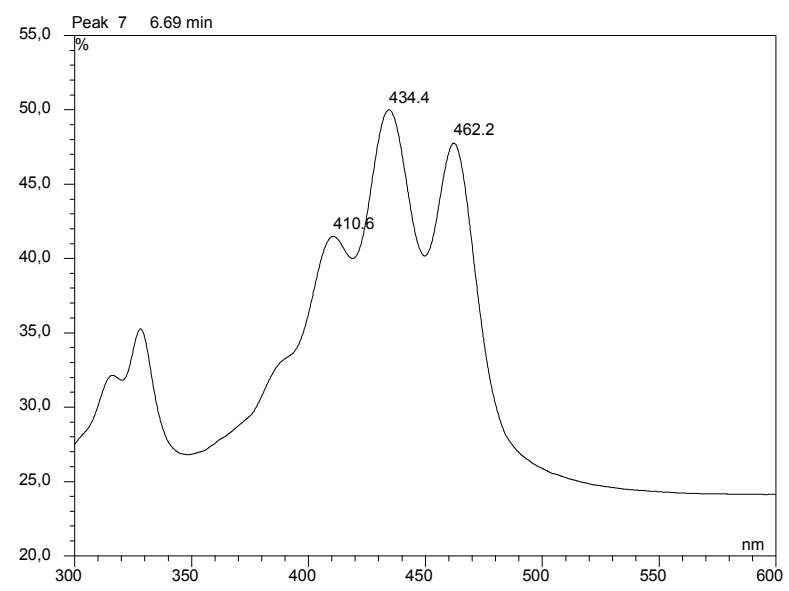

Peak 8: (all-E)-Neoxanthin

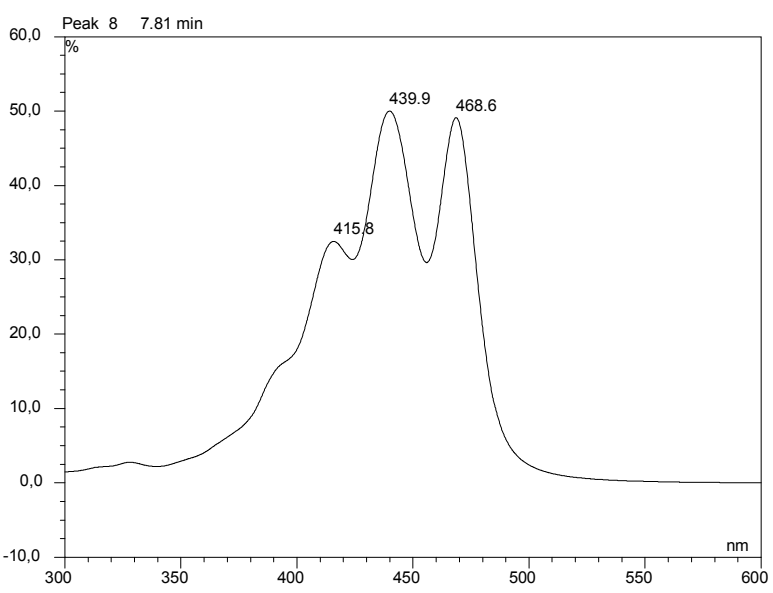

\section{Peak 9: Neochrome}

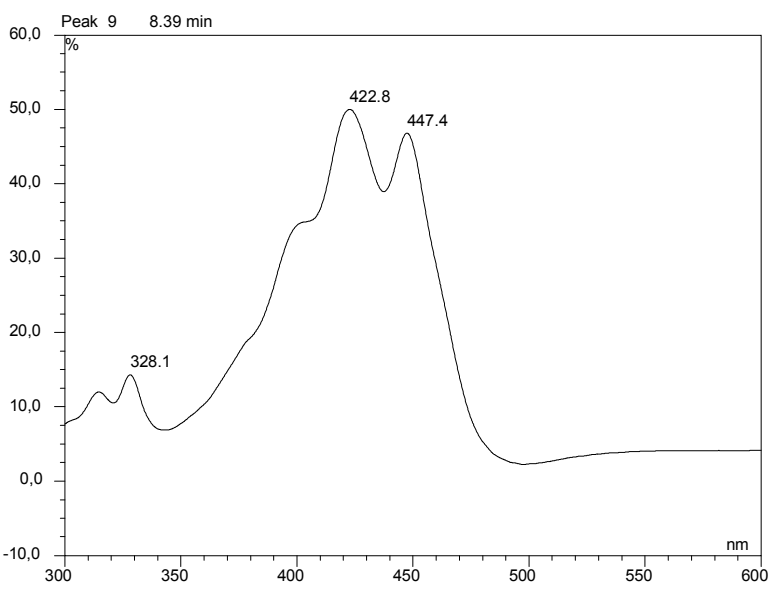




\section{Peak 10: Violaxanthin}

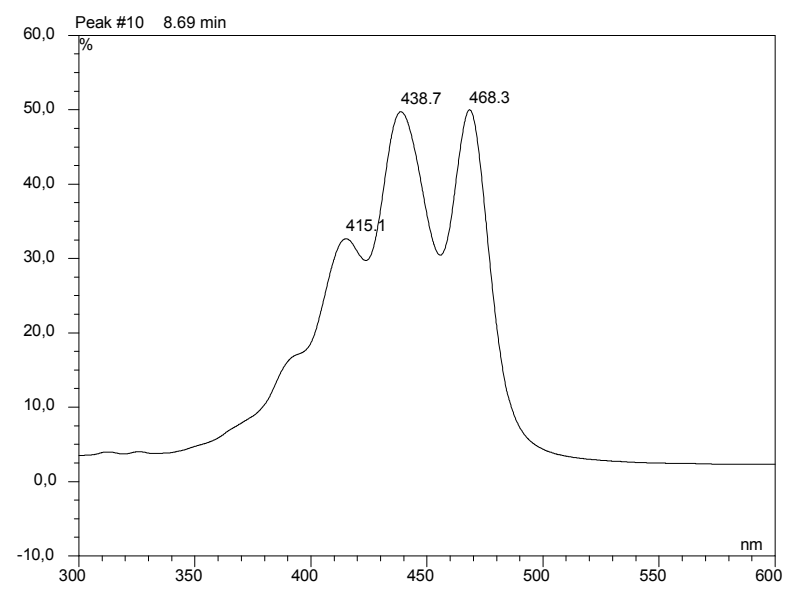

Peak 11: Unidentified 5,8-epoxide

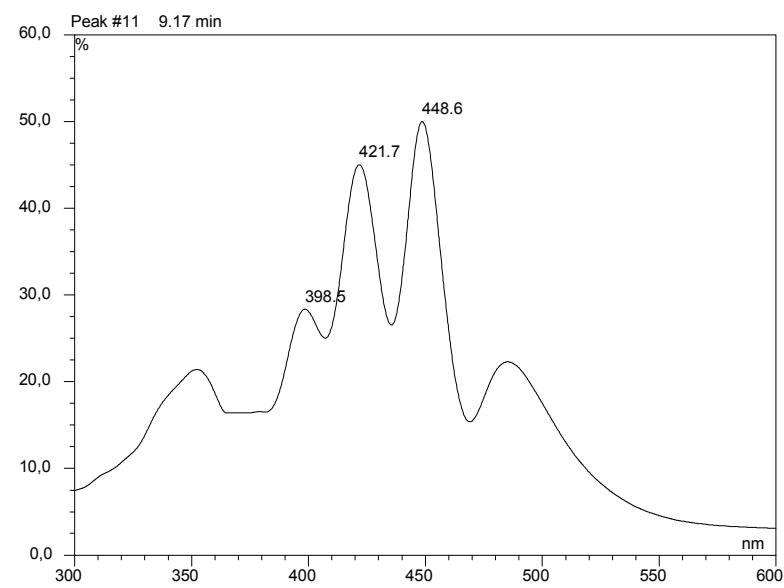

Peak 12: Capsoneoxanthin

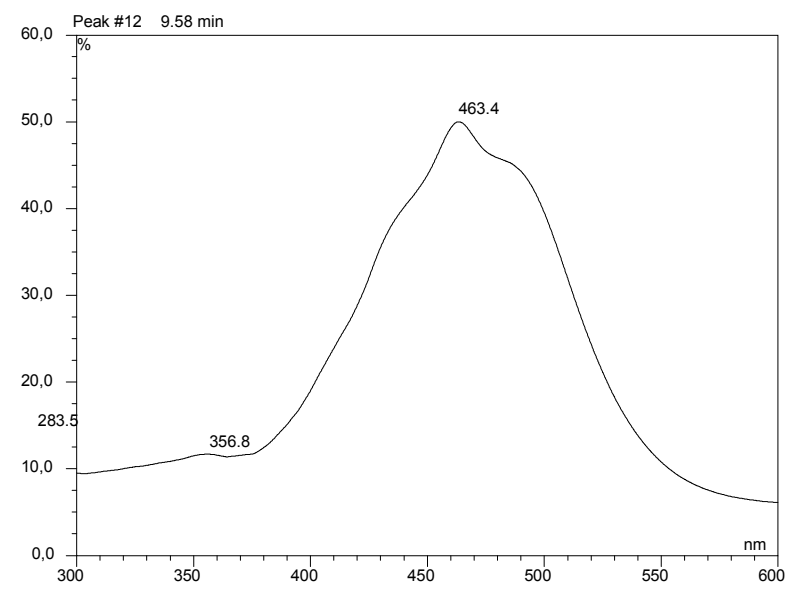




\section{Peak 13: Luteoxanthin}

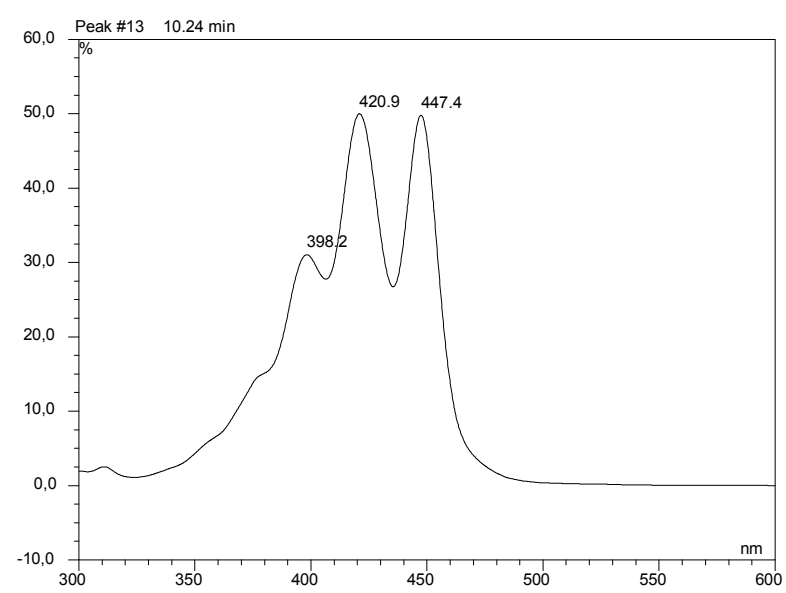

Peak 14: Capsanthin 5,6-epoxide

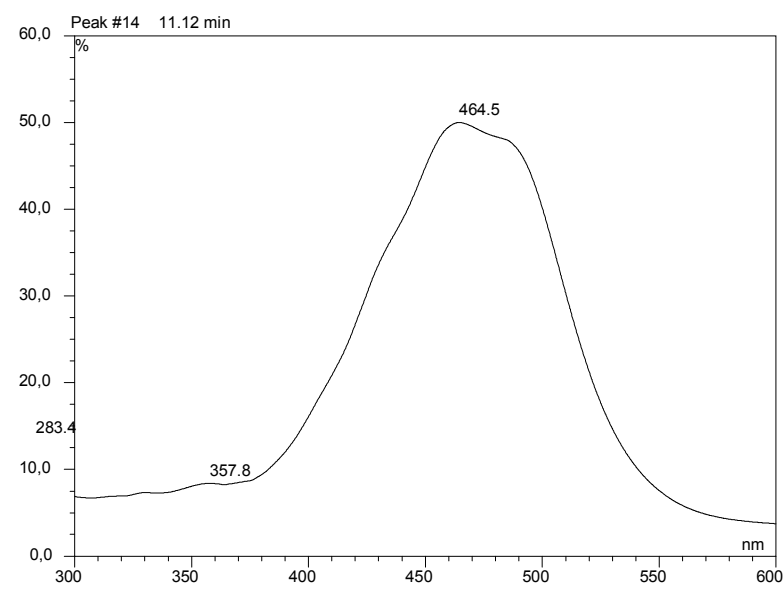

Peak 15: $\beta$-Cryptoxanthin 5,6,5',6'-epoxide

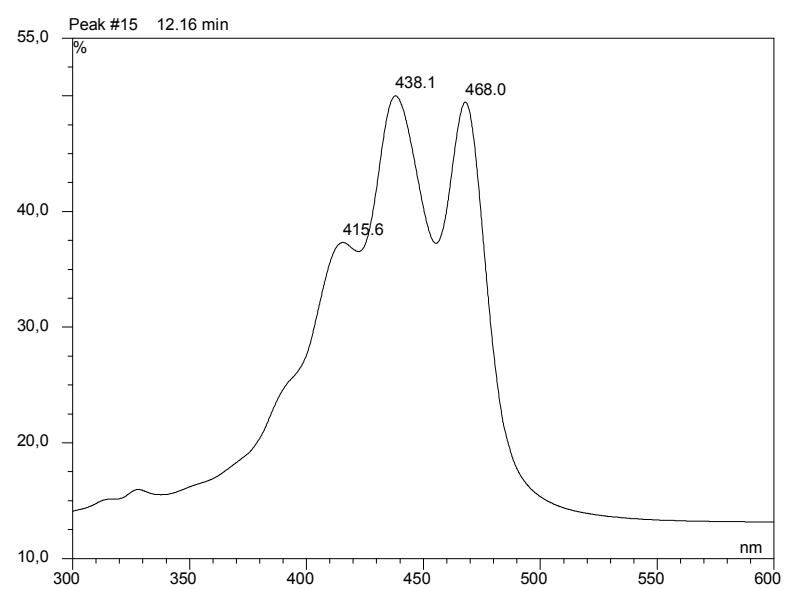




\section{Peak 16: (9Z)-Violaxanthin}

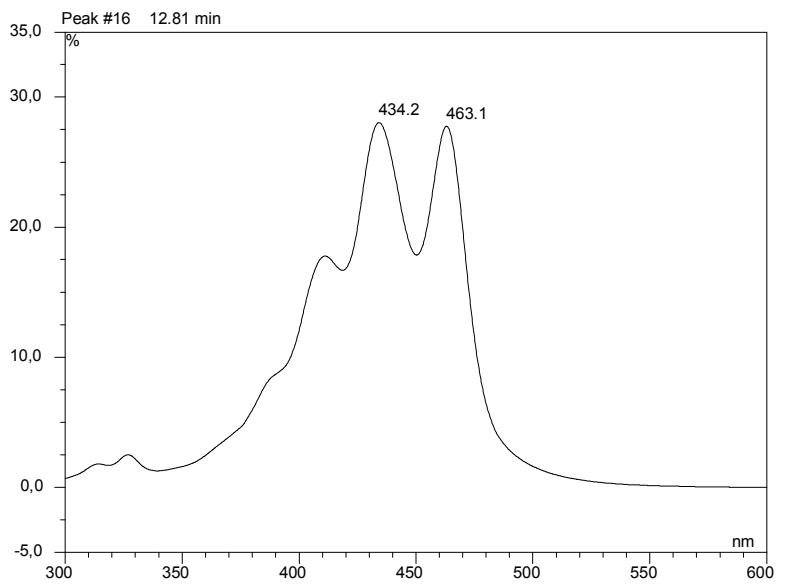

Peak 17: Unidentified

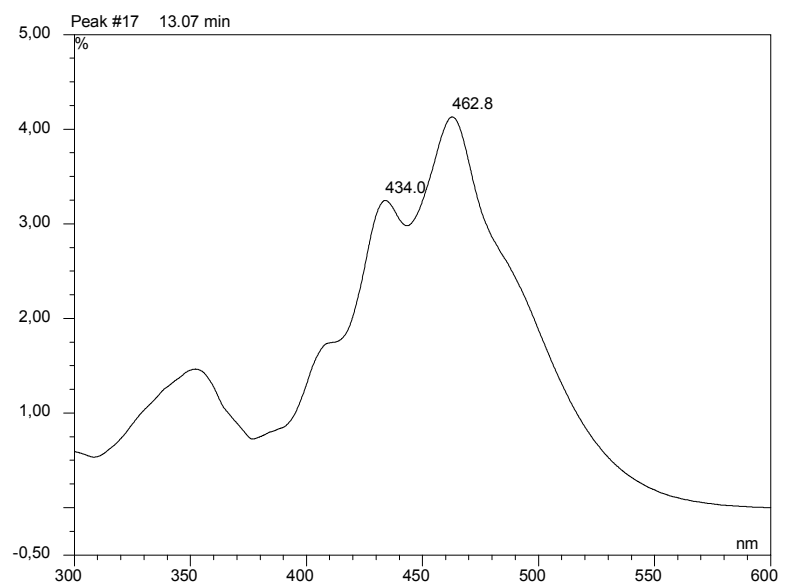

Peak 18: Unidentified 5,8-epoxide

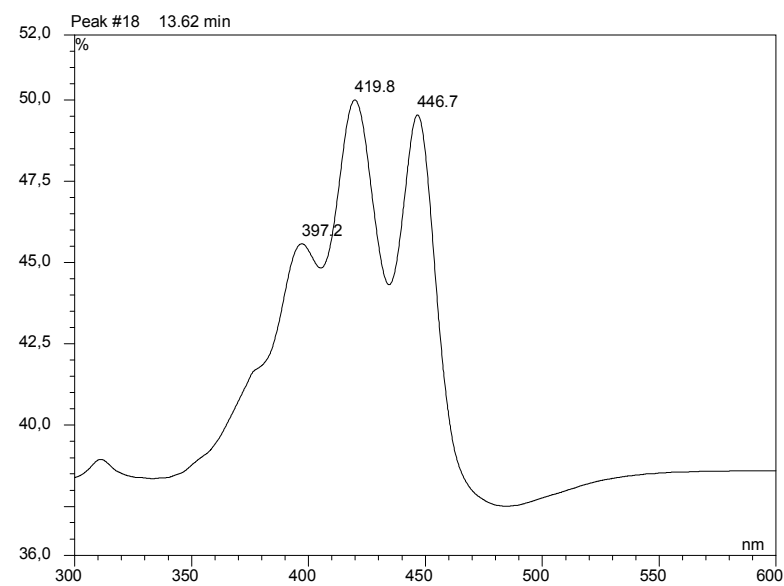


Peak 19: $\beta$-Cryptoxanthin 5,6,5',6'-diepoxide

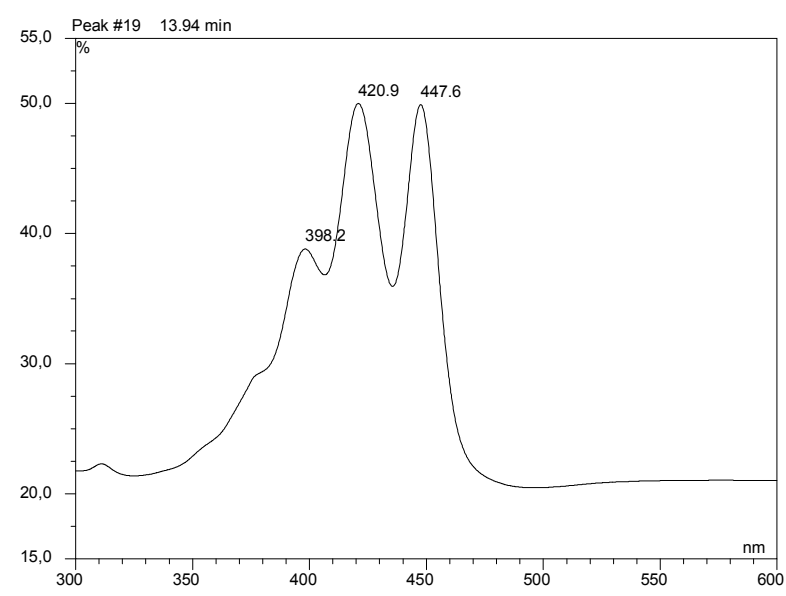

Peak 20: $\beta$-Cryptoxanthin 5,8,5',6'-diepoxide

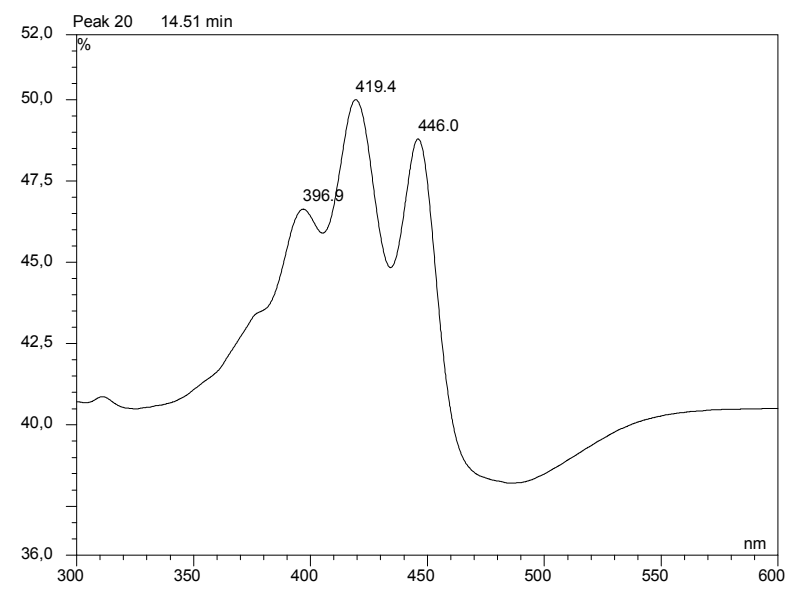

Peak 21: Capsorubin + Unidentified

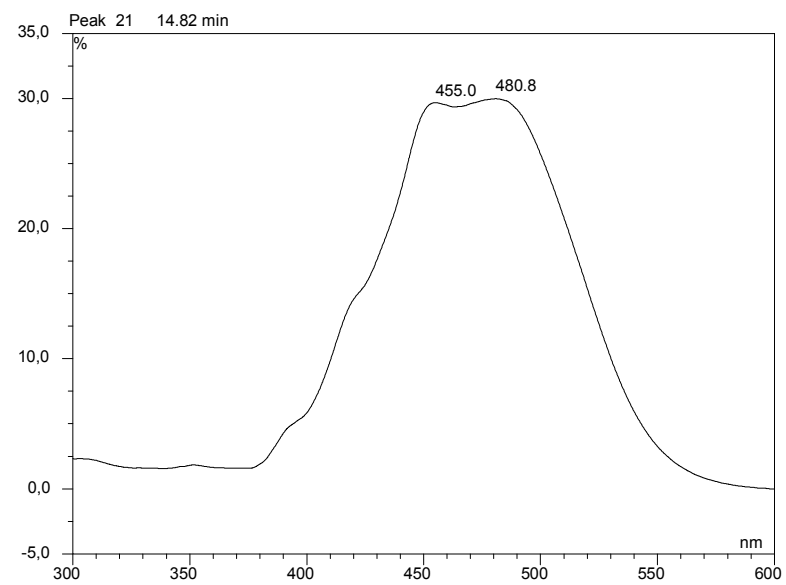


Peak 22: Cryptocapsin 5,6-epoxide

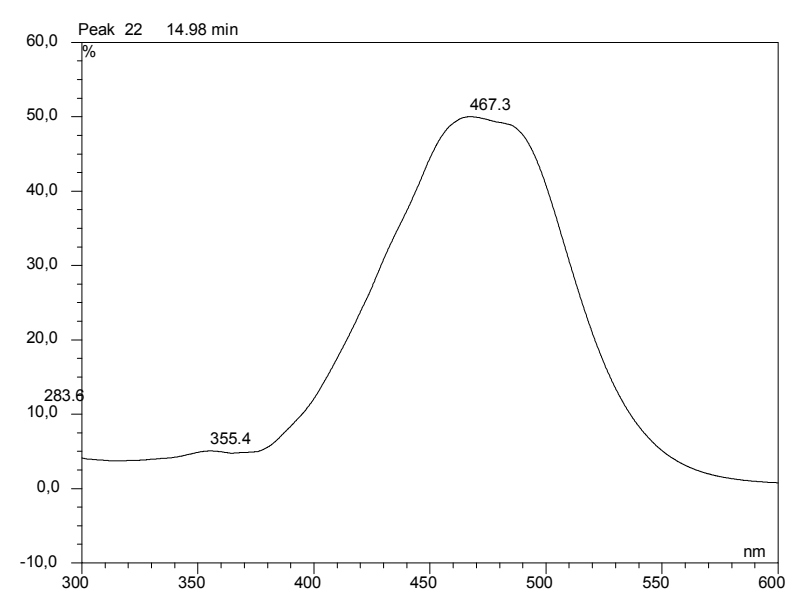

Peak 23: 3'-Deoxycapsanthin 5,6-epoxide + unidentified

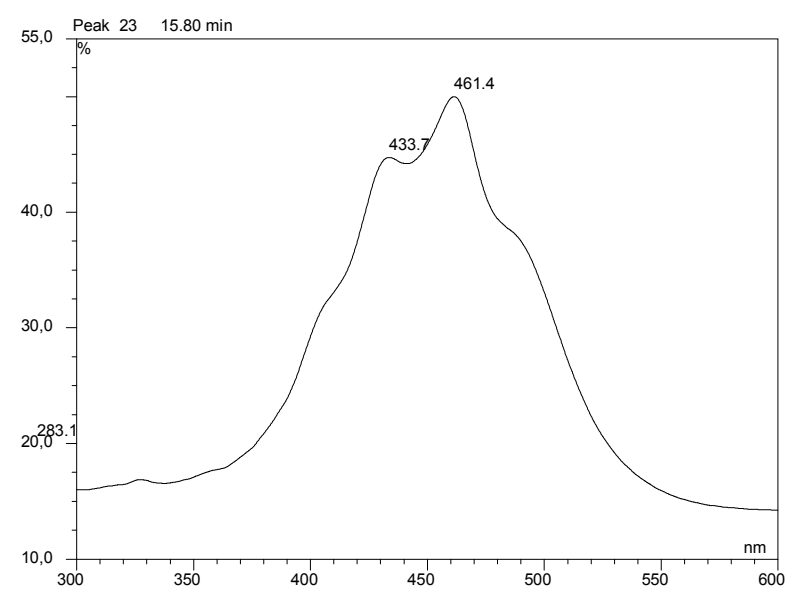

Peak 24: $\beta$-Cryptoxanthin 5,8,5',8'-diepoxide + (9Z)-Capsanthin 5,6-epoxide

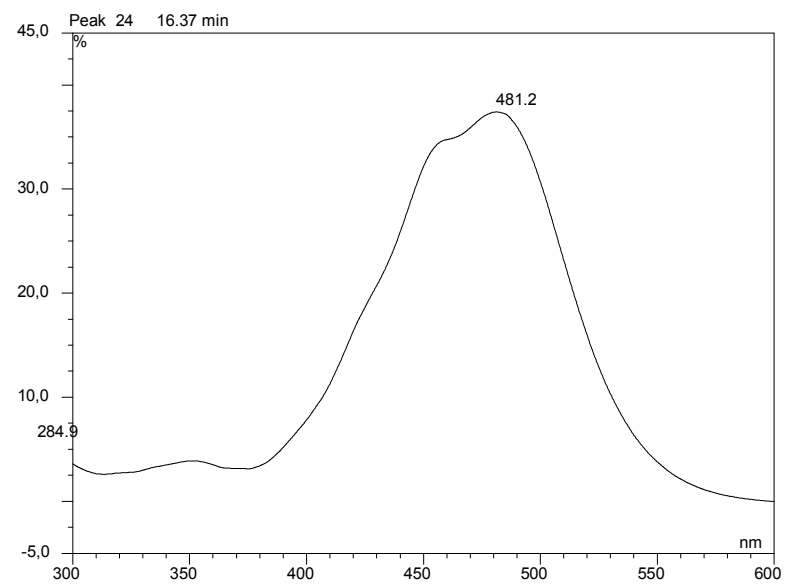


Peak 27: (13/13’Z)-Unidentified

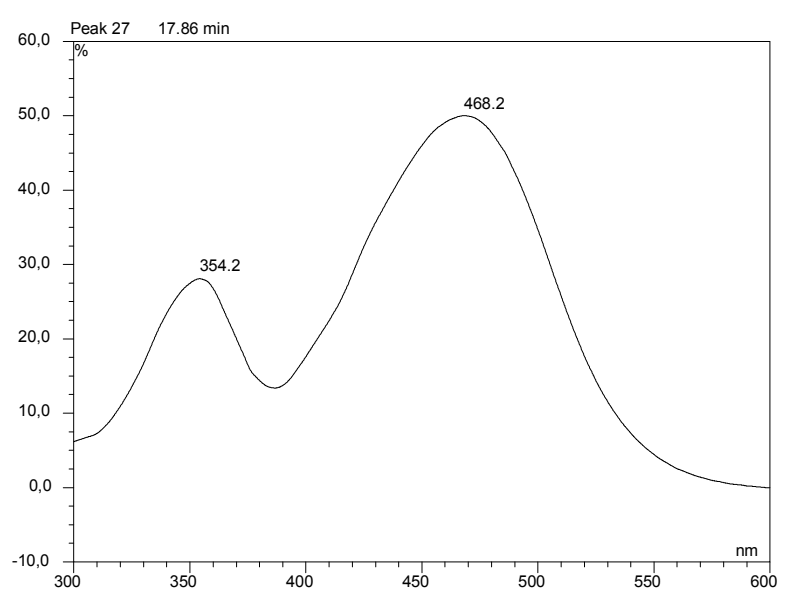

Peak 28: Unidentified

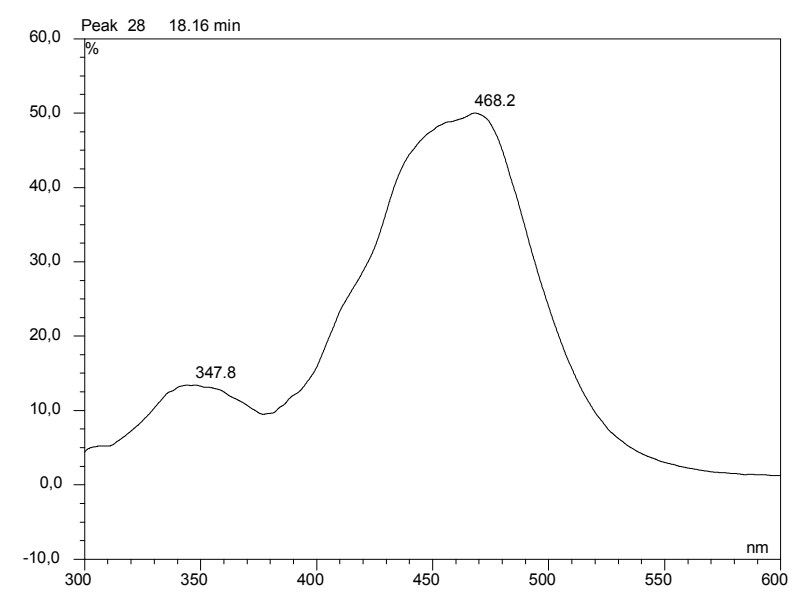

Peak 29: $\beta$-Carotene 5,6,5',8'-epoxide

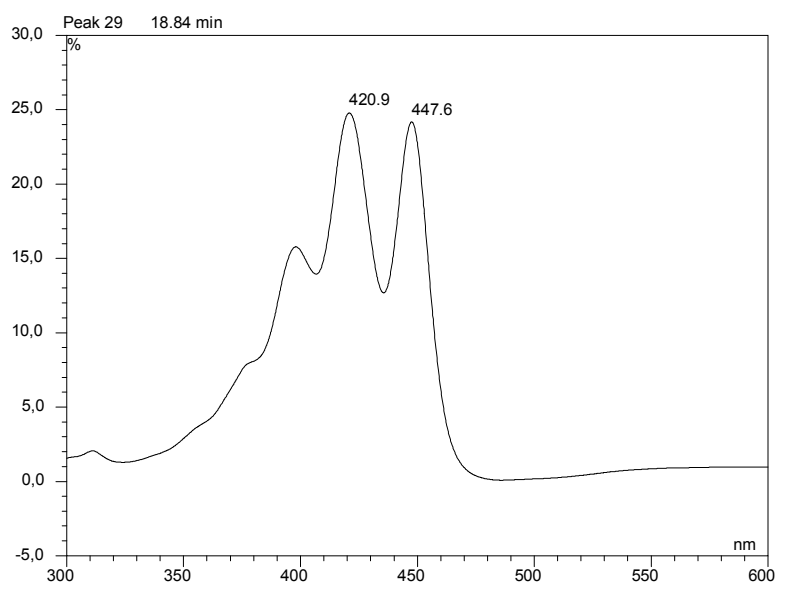




\section{Peak 30: (13/13'Z)-Cryptocapsin}

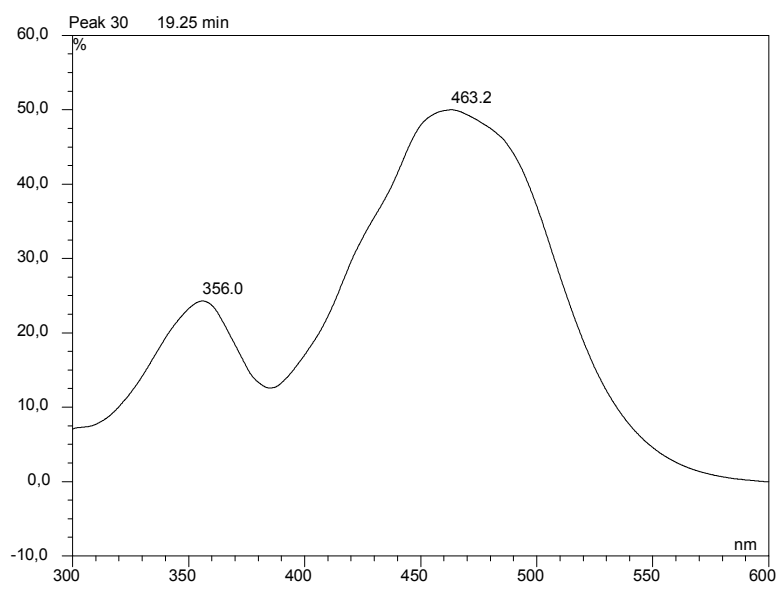

\section{Peak 31: (13'/13Z)-Cryptocapsin}

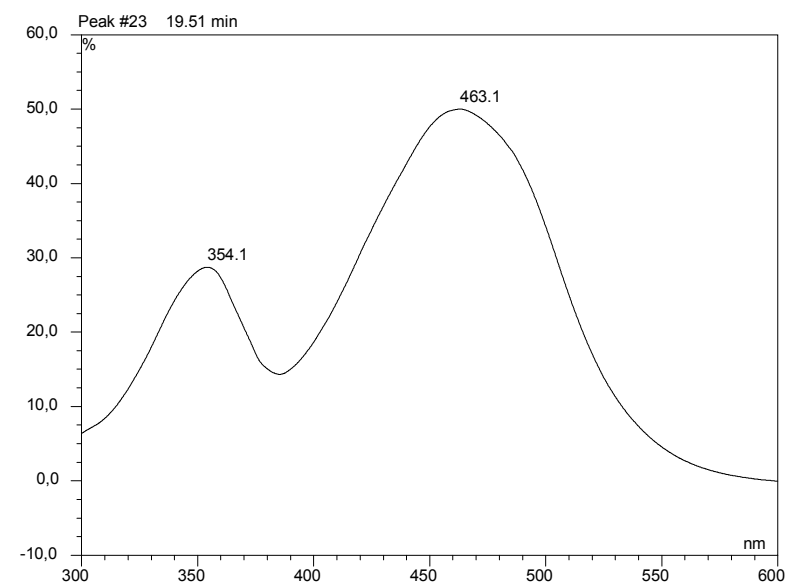

Peak 32: $\beta$-Cryptoxanthin 5,6-epoxide

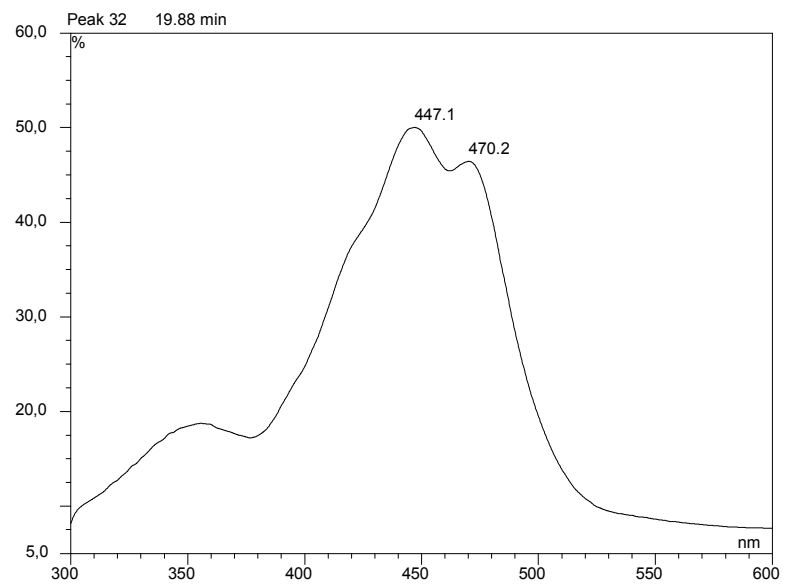




\section{Peak 33: Unidentified mixture}

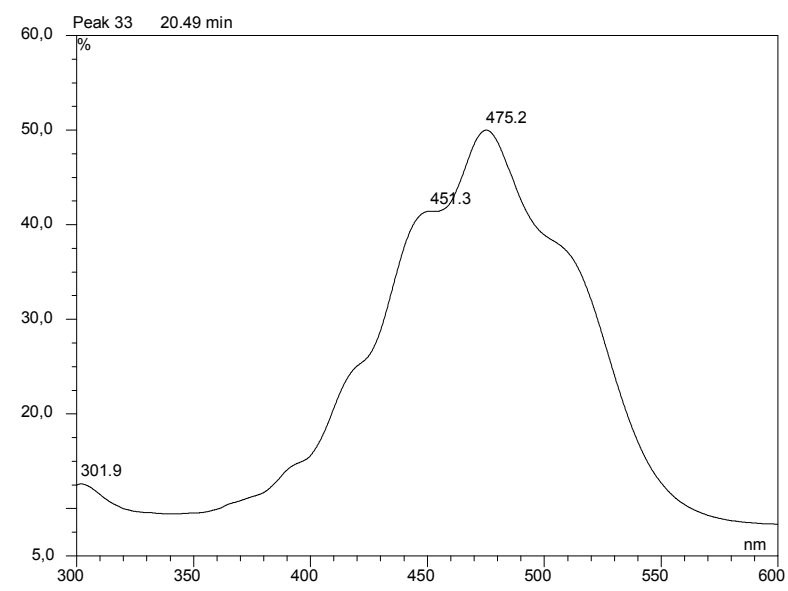

Peak 35: 3'-Deoxycapsanthin

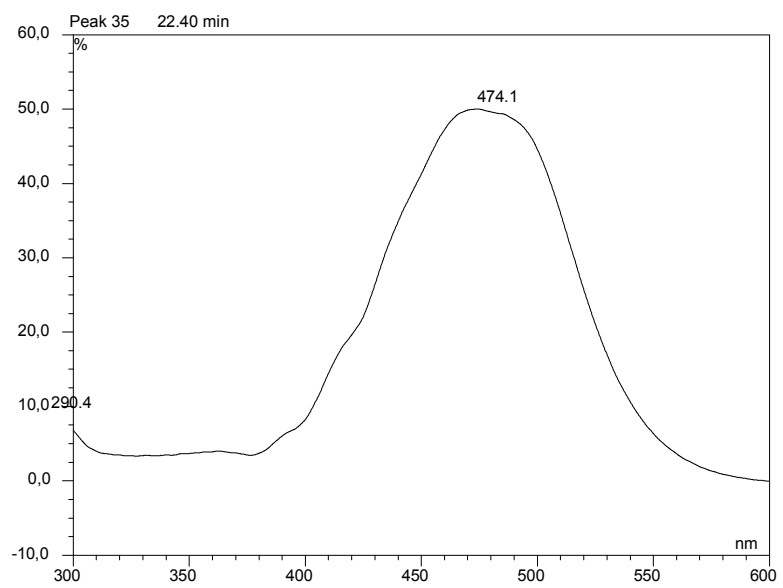

Peak 36: Unidentified

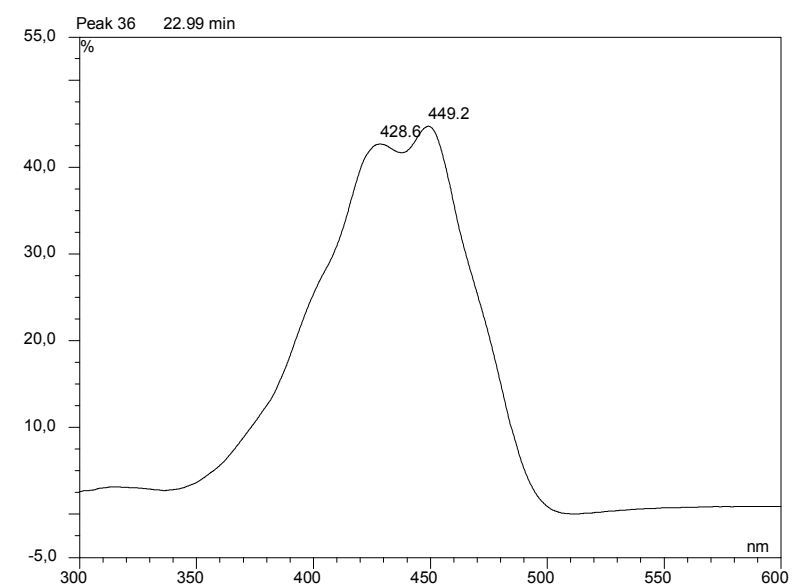


Peak 37: $\beta$-Cryptoxanthin 5,8-epoxide

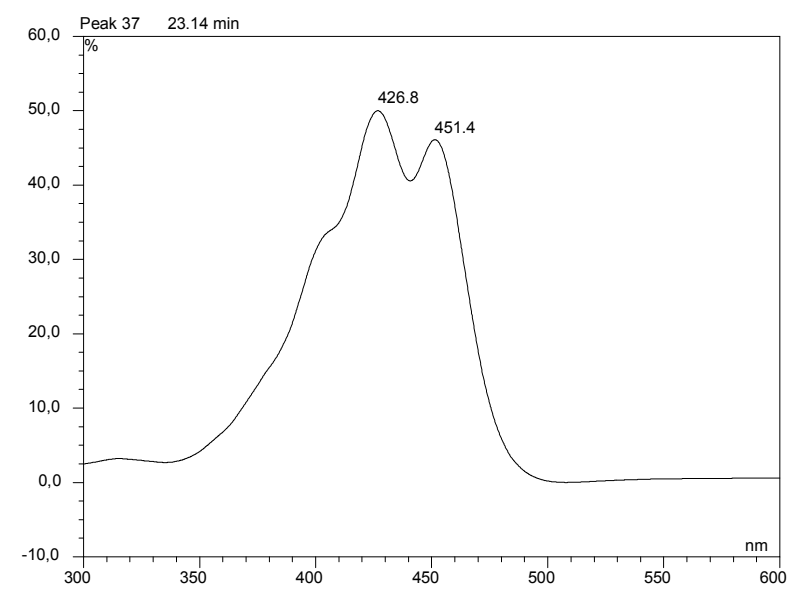

Peak 38: $\beta$-Cryptoxanthin 5',8'-epoxide

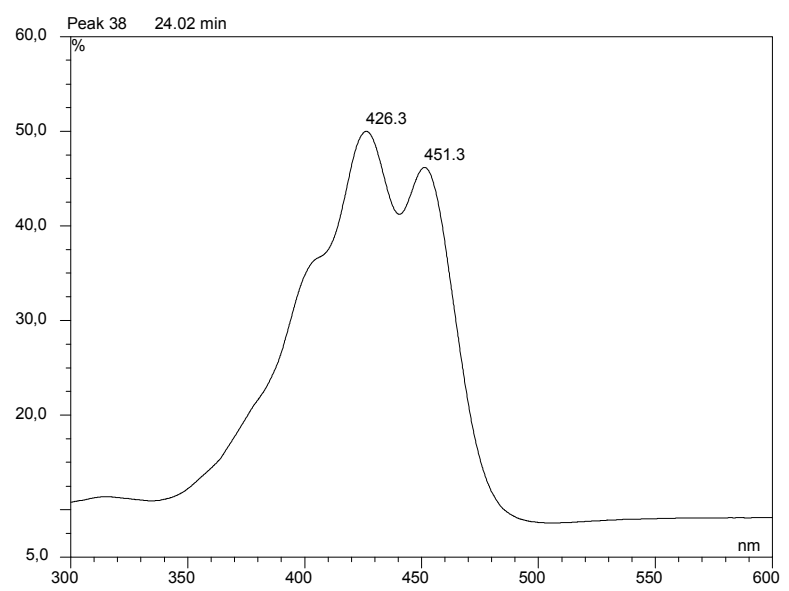

Peak 39: Unidentified

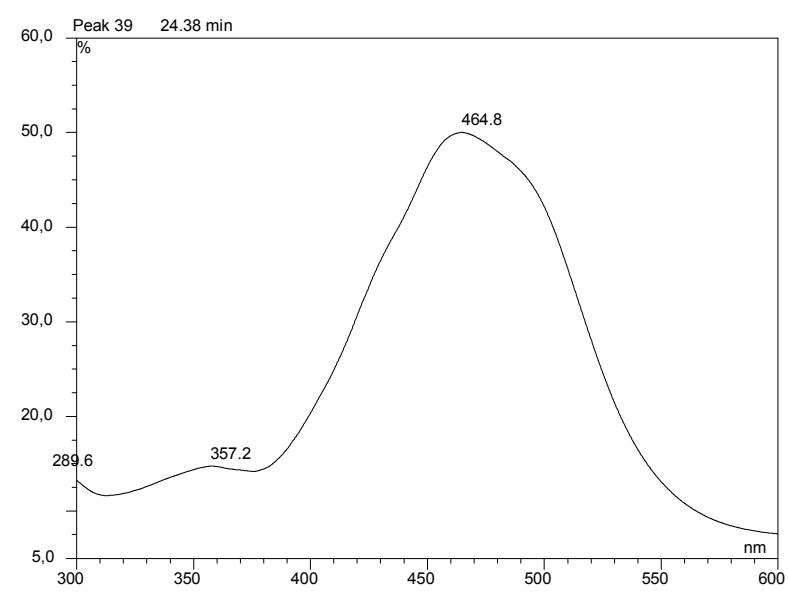




\section{Peak 40: Cryptocapsin}

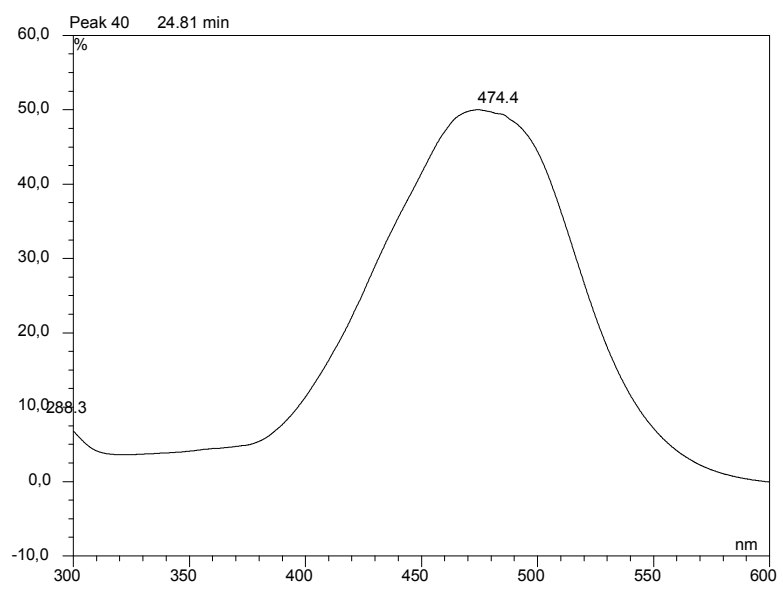

Peak 41: (13/13'Z)-Sapotexanthin

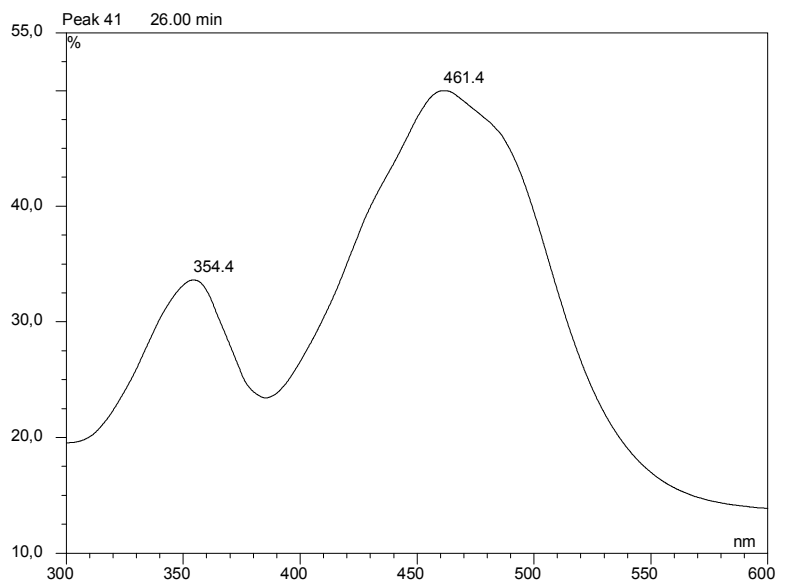

Peak 42: $\beta$-Carotene 5,6-epoxide

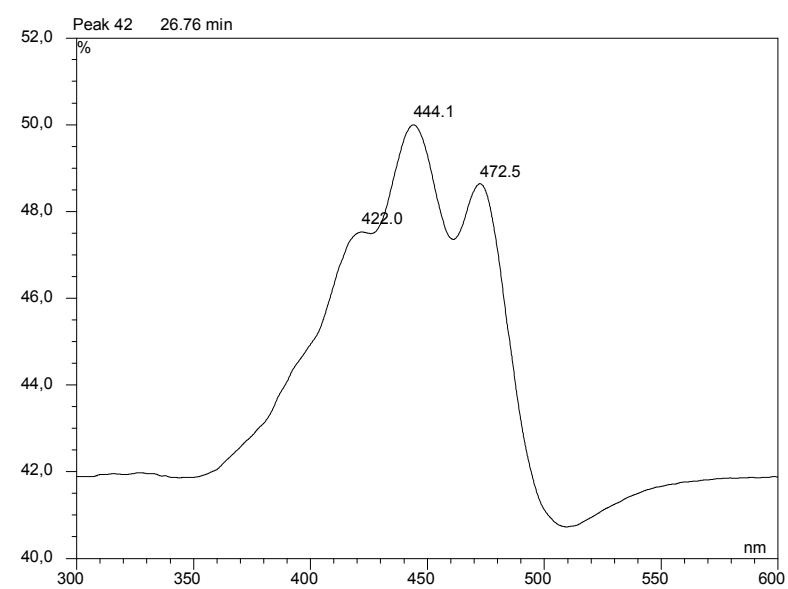


Peak 44: $\beta$-Carotene 5,8-epoxide

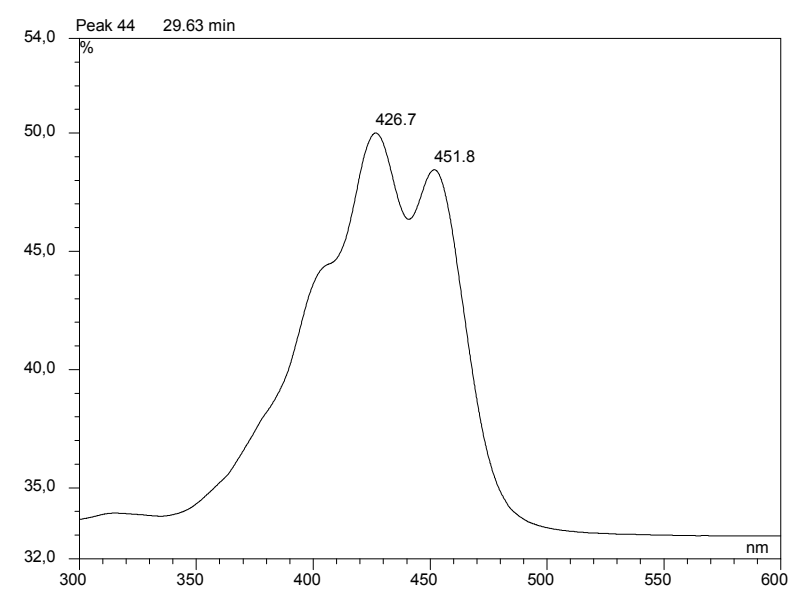

\section{Peak 45: Sapotexanthin}

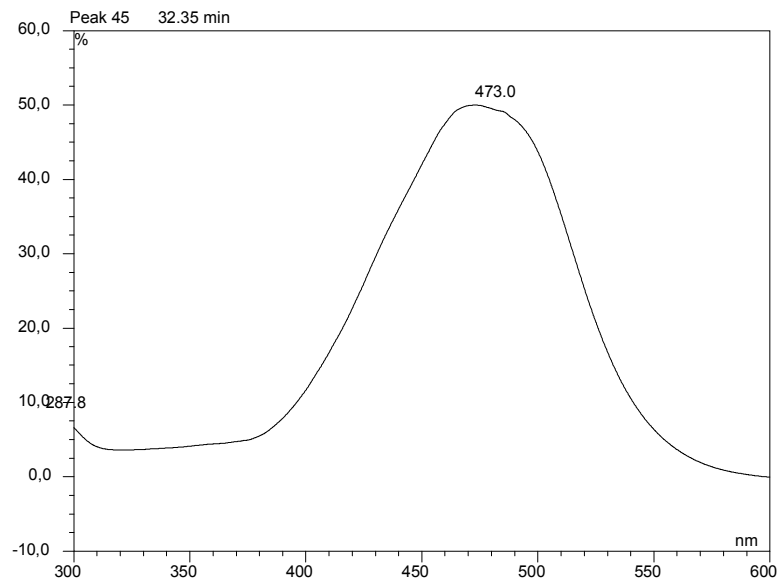

Peak 47: $\beta$-Carotene

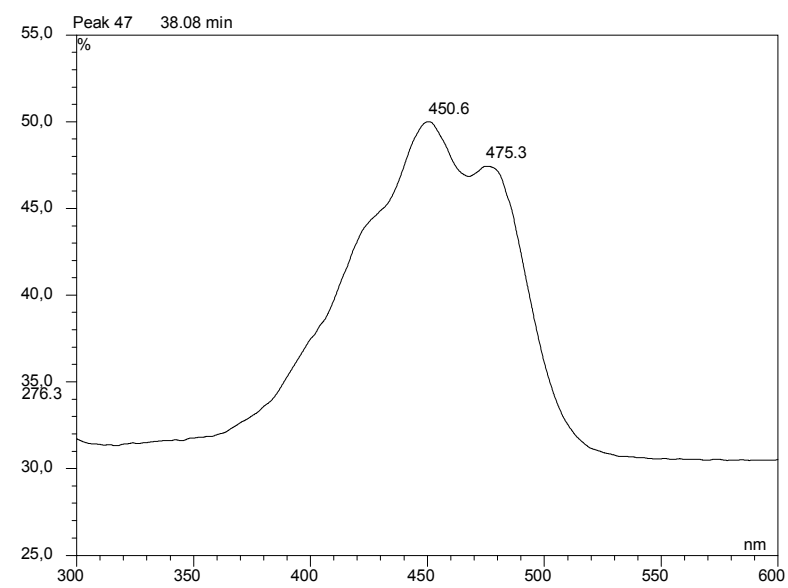

